\title{
Standards of Medical Care in Diabetes-2018 Abridged for Primary Care Providers
}

American Diabetes Association

This is an abridged version of the American Diabetes Association's Standards of Medical Care in Diabetes-2018. Diabetes Care 2018;41(Suppl. 1):S1-S159.

The complete 2018 Standards supplement, including all supporting references, is available at professional.diabetes.org/ standards.

https://doi.org/10.2337/cd17-0119

(C)2018 by the American Diabetes Association. Readers may use this article as long as the work is properly cited, the use is educational and not for profit, and the work is not altered. See http:// creativecommons.org/licenses/by-nc-nd/3.0 for details.
I he American Diabetes Association's (ADA's) Standards of Medical Care in Diabetes are published each year in a supplement to the January issue of Diabetes Care. The ADA's Professional Practice Committee develops the Standards and updates them annually, or more frequently online should it determine that new evidence or regulatory changes (e.g., drug approvals, label changes) merit immediate incorporation. The Standards include the most current evidence-based recommendations for diagnosing and treating adults and children with diabetes. ADA's grading system uses $A, B, C$, or $E$ to show the evidence level that supports each recommendation.

- A-Clear evidence from wellconducted, generalizable randomized controlled trials that are adequately powered

- B-Supportive evidence from well-conducted cohort studies

- C-Supportive evidence from poorly controlled or uncontrolled studies

- E-Expert consensus or clinical experience

This is an abridged version of the Standards containing the evidence-based recommendations most pertinent to primary care. The tables and figures have been renumbered from the original document to match this version. All of the recommendations (bulleted text) are precisely the same as in the full Standards of Care. The complete 2018 Standards of Care document, including all supporting references, is available at professional. diabetes.org/standards.

\section{IMPROVING CARE AND PROMOTING HEALTH IN POPULATIONS}

Over the past 10 years, the proportion of patients with diabetes who achieve recommended $\mathrm{A} 1 \mathrm{C}$, blood pressure, and LDL cholesterol levels has increased. The mean A1C nationally among people with diabetes has declined from $7.6 \%(60 \mathrm{mmol} / \mathrm{mol})$ in $1999-2002$ to $7.2 \%(55 \mathrm{mmol} / \mathrm{mol})$ in 2007-2010 based on the National Health and Nutrition Examination Survey, with younger adults less likely to meet treatment targets than older adults. This has been accompanied by improvements in cardiovascular outcomes and has led to substantial reductions in end-stage microvascular complications. Nevertheless, 33-49\% of patients still do not meet targets for glycemic, blood pressure, or cholesterol control, and only 14\% meet targets for all three measures while also avoiding smoking.

Optimal diabetes management requires an organized, systematic approach and the involvement of a coordinated team of dedicated health care professionals working in an environment where patient-centered high-quality care is a priority.

\section{Recommendations}

- Ensure treatment decisions are timely, rely on evidence-based guidelines, and are made collaboratively with patients based on 
individual preferences, prognoses, and comorbidities. B

- Align approaches to diabetes management with the Chronic Care Model, emphasizing productive interactions between a prepared proactive care team and an informed activated patient. A

- Care systems should facilitate team-based care, patient registries, decision support tools, and community involvement to meet patient needs. B

- Efforts to assess the quality of diabetes care and create quality improvement strategies should incorporate reliable data metrics, to promote improved processes of care and health outcomes, with simultaneous emphasis on costs. E

\section{Tailoring Treatment for Social Context}

Health inequities related to diabetes and its complications are well documented and are heavily influenced by social determinants of health. Social determinants of health are defined as the economic, environmental, political, and social conditions in which people live and are responsible for a major part of health inequality worldwide. The ADA recognizes the association between social and environmental factors and risk for diabetes and its complications.

\section{Recommendations}

- Providers should assess social context, including potential food insecurity, housing stability, and financial barriers, and apply that information to treatment decisions. A

- Refer patients to local community resources when available. $B$

- Provide patients with self-management support from lay health coaches, navigators, or community health workers when available. A

\section{CLASSIFICATION AND DIAGNOSIS OF DIABETES}

Diabetes can be classified into the following general categories:

1. Type 1 diabetes (due to autoim- mune $\beta$-cell destruction, usually leading to absolute insulin deficiency)

2. Type 2 diabetes (due to a progressive loss of $\beta$-cell insulin secretion frequently on the background of insulin resistance)

3. Gestational diabetes mellitus (GDM) (diabetes diagnosed in the second or third trimester of pregnancy that was not clearly overt diabetes prior to gestation)

4. Specific types of diabetes due to other causes, e.g., monogenic diabetes syndromes (such as neonatal diabetes and maturity-onset diabetes of the young), diseases of the exocrine pancreas (such as cystic fibrosis and pancreatitis), and drug- or chemical-induced diabetes (such as with glucocorticoid use, in the treatment of HIV/AIDS, or after organ transplantation)

\section{Diagnostic Tests for Diabetes}

Diabetes and prediabetes may be screened based on plasma glucose criteria, either the fasting plasma glucose (FPG) or the 2-h plasma glucose (2-h PG) value during a 75-g oral glucose tolerance test (OGTT), or A1C criteria (Table 1).

There is incomplete concordance between A1C, FPG, and 2-h PG, and the 2-h PG diagnoses more people with diabetes than the FPG or A1C. Marked discrepancies between measured A1C and plasma glucose levels should prompt consideration that the A1C assay may not be reliable for that individual, since a relatively small percentage of patients have conditions such as sickle cell trait or hemoglobinopathies that skew A1C results. See the full 2018 Standards of Care for conditions causing discrepancies. Unless there is a clear clinical diagnosis based on overt signs of hyperglycemia, a second test is required for confirmation, either repeating the same test used initially or a different test. If patients have test results near the margins of the diagnostic threshold, the health care professional should follow the patient closely and repeat the test in 3-6 months.

\section{Categories of Increased Risk for Diabetes (Prediabetes)}

"Prediabetes" is the term used for individuals whose glucose levels do not meet the criteria for diabetes but are too high to be considered normal (see Table 1). Prediabetes should not be viewed as a clinical entity in its own right but rather as an increased risk for diabetes and cardiovascular disease (CVD).

\section{Recommendations}

- Screening for prediabetes and risk for future diabetes with an informal assessment of risk factors or validated tools should be considered in asymptomatic adults. B

- Testing for prediabetes and risk for future diabetes in asymptomatic people should be considered in adults of any age who are overweight or obese (BMI $\geq 25 \mathrm{~kg} / \mathrm{m}^{2}$ or $\geq 23 \mathrm{~kg} / \mathrm{m}^{2}$ in Asian Americans) and who have one or more addi-

TABLE 1. Criteria for the Screening and Diagnosis of Diabetes

\begin{tabular}{lcc}
\hline & Prediabetes & Diabetes \\
\hline A1C & $5.7-6.4 \% *$ & $\geq 6.5 \%+$ \\
\hline FPG & $100-125 \mathrm{mg} / \mathrm{dL}(5.6-6.9 \mathrm{mmol} / \mathrm{L})^{*}$ & $\geq 126 \mathrm{mg} / \mathrm{dL}(7.0 \mathrm{mmol} / \mathrm{L}) \dagger$ \\
\hline OGTT & $140-199 \mathrm{mg} / \mathrm{dL}(7.8-11.0 \mathrm{mmol} / \mathrm{L})^{*}$ & $\geq 200 \mathrm{mg} / \mathrm{dL}(11.1 \mathrm{mmol} / \mathrm{L}) \dagger$ \\
\hline RPG & - & $\geq 200 \mathrm{mg} / \mathrm{dL}(11.1 \mathrm{mmol} / \mathrm{L}) \neq$ \\
\hline
\end{tabular}

*For all three tests, risk is continuous, extending below the lower limit of the range and becoming disproportionately greater at the higher end of the range. tIn the absence of unequivocal hyperglycemia, results should be confirmed by repeat testing. $¥$ Only diagnostic in a patient with classic symptoms of hyperglycemia or hyperglycemic crisis. RPG, random plasma glucose. 


\section{TABLE 2. Criteria for Testing for Diabetes or Prediabetes} in Asymptomatic Adults

1. Testing should be considered in overweight or obese (BMI $\geq 25 \mathrm{~kg} / \mathrm{m}^{2}$ or $\geq 23 \mathrm{~kg} / \mathrm{m}^{2}$ in Asian Americans) adults who have one or more of the following risk factors:

- First-degree relative with diabetes

- High-risk race/ethnicity (e.g., African American, Latino, Native American, Asian American, Pacific Islander)

- History of CVD

- Hypertension ( $\geq 140 / 90 \mathrm{mmHg}$ or on therapy for hypertension)

- HDL cholesterol level $<35 \mathrm{mg} / \mathrm{dL}(0.90 \mathrm{mmol} / \mathrm{L})$ and/or a triglyceride level $>250 \mathrm{mg} / \mathrm{dL}(2.82 \mathrm{mmol} / \mathrm{L})$

- Women with polycystic ovary syndrome

- Physical inactivity

- Other clinical conditions associated with insulin resistance (e.g., severe obesity, acanthosis nigricans)

2. Patients with prediabetes (A1C $\geq 5.7 \%$ [39 $\mathrm{mmol} / \mathrm{mol}], \mathrm{IGT}$, or IFG) should be tested yearly.

3. Women who were diagnosed with GDM should have lifelong testing at least every 3 years.

4. For all other patients, testing should begin at age 45 years.

5. If results are normal, testing should be repeated at a minimum of 3-year intervals, with consideration of more frequent testing depending on initial results and risk status.

TABLE 3. Risk-Based Screening for Type 2 Diabetes or Prediabetes in Asymptomatic Children and Adolescents in a Clinical Setting*

Criteria

- $\quad$ Overweight (BMI >85th percentile for age and sex, weight for height $>85$ th percentile, or weight $>120 \%$ of ideal for height) $A$

Plus one or more additional risk factors based on the strength of their association with diabetes as indicated by evidence grades:

- Maternal history of diabetes or GDM during the child's gestation A

- Family history of type 2 diabetes in first- or second-degree relative A

- Race/ethnicity (Native American, African American, Latino, Asian American, Pacific Islander) A

- Signs of insulin resistance or conditions associated with insulin resistance (acanthosis nigricans, hypertension, dyslipidemia, polycystic ovary syndrome, or small-for-gestational-age birth weight) B

*Persons aged $<18$ years.

tional risk factors for diabetes (Table 2). B

- For all people, testing should begin at age 45 years. $B$

- If tests are normal, repeat testing carried out at a minimum of 3 -year intervals is reasonable. $\mathrm{C}$

- To test for prediabetes, FPG, 2-h PG during 75-g OGTT tolerance test, and A1C are equally appropriate. $B$

- In patients with prediabetes, identify and, if appropriate, treat other CVD risk factors. $\mathrm{B}$

- Testing for prediabetes should be considered in children and adolescents who are overweight or obese (BMI >85th percentile for age and sex, weight for height $>85$ th percentile, or weight $>120 \%$ of ideal for height) and who have additional risk factors for diabetes (Table 3). E

\section{COMPREHENSIVE \\ MEDICAL EVALUATION AND ASSESSMENT OF COMORBIDITIES}

\section{Patient-Centered Collaborative Care}

\section{Recommendation}

- A patient-centered communication style that uses person-centered and strength-based language, uses active listening, elicits patient preferences and beliefs, and assesses literacy, numeracy, and potential barriers to care should be used to optimize patient health outcomes and health-related quality of life. B

\section{Comprehensive Medical Evaluation}

\section{Recommendations}

- A complete medical evaluation should be performed at the initial visit to:

- Confirm the diagnosis and classify diabetes. B

- Evaluate for diabetes complications and potential comorbid conditions. E

- Begin patient engagement in the formulation of a care management plan. B

- Develop a plan for continuing care. B

- A follow-up visit should include most components of the initial comprehensive medical evaluation including: interval medical history; assessment of medicationtaking behavior and intolerance/ side effects; physical examination; laboratory evaluation as appropriate to assess attainment of $\mathrm{A} 1 \mathrm{C}$ and metabolic targets; and assessment of risk for complications, diabetes self-management behaviors, nutrition, psychosocial health, and the need for referrals, immunizations, or other routine health maintenance screening. $B$ 
Table 4 lists the components of the diabetes medical evaluation.

\section{Immunization}

Children and adults with diabetes should receive vaccinations according to age-specific recommendations. See the Centers for Disease Control and Prevention website for current recommendations.

\section{Assessment of Comorbidities}

Besides assessing diabetes-related complications, clinicians and their patients need to be aware of common comorbidities that affect people with diabetes and may complicate management.

\section{Autoimmune Diseases}

\section{Recommendation}

- Consider screening patients with type 1 diabetes for autoimmune thyroid disease and celiac disease soon after diagnosis. B

\section{Cognitive Impairment/ Dementia}

See Psychosocial Care and Older Adults sections.

\section{Recommendation}

- In people with a history of cognitive impairment/dementia, intensive glucose control cannot be expected to remediate deficits. Treatment should be tailored to avoid significant hypoglycemia. B

Other conditions, including fatty liver disease, hepatocellular carcinoma, hip fractures, low testosterone in men, obstructive sleep apnea, and periodontal disease are all more common in persons with diabetes. See the full 2018 Standards of Care for discussion on these topics.

\section{Cancer}

Diabetes is associated with increased risk of cancers of the liver, pancreas, endometrium, colon/rectum, breast, and bladder. The association may result from shared risk factors between type 2 diabetes and cancer (older age, obesity, and physical inactivity) but may also be due to diabetes-related factors, such as underlying disease physiology or diabetes treatments. Patients with diabetes should be encouraged to undergo recommended age- and sex-appropriate cancer screenings and to reduce their modifiable cancer risk factors (obesity, physical inactivity, and smoking).

\section{LIFESTYLE MANAGEMENT}

Lifestyle management is a fundamental aspect of diabetes care and includes diabetes self-management education and support (DSMES), medical nutrition therapy (MNT), physical activity, smoking cessation counseling, and psychosocial care. Patients and care providers should focus together on how to optimize lifestyle from the time of the initial comprehensive medical evaluation, throughout all subsequent evaluations and follow-up, and during the assessment of complications and management of comorbid conditions in order to enhance diabetes care.

\section{DSMES}

\section{Recommendations}

- In accordance with the national standards for DSMES, all people with diabetes should participate in diabetes self-management education to facilitate the knowledge, skills, and ability necessary for diabetes self-care and in diabetes self-management support to assist with implementing and sustaining skills and behaviors needed for ongoing self-management. B

- There are four critical times to evaluate the need for DSMES: at diagnosis, annually, when complicating factors arise, and when transitions in care occur. E

- Facilitating appropriate diabetes self-management and improving clinical outcomes, health status, and quality of life are key goals of DSMES to be measured and monitored as part of routine care. C

- Effective DSMES should be patient centered, may be given in group or individual settings or using technology, and should help guide clinical decisions. A
- Because DSMES can improve outcomes and reduce costs B, adequate reimbursement by thirdparty payers is recommended. $\mathrm{E}$

\section{Nutrition Therapy}

For a complete discussion and references, see the ADA position statement "Nutrition Therapy Recommendations for the Management of Adults With Diabetes."

Goals of nutrition therapy for adults with diabetes are to promote and support healthful eating patterns in achieving and maintaining body weight, glycemic, blood pressure, and lipid goals while addressing individual issues, including access to healthful foods, personal and cultural preferences, and other factors.

Evidence suggests that there is not an ideal percentage of calories from carbohydrate, protein, and fat for all people with diabetes. Therefore, macronutrient distribution should be based on an individualized assessment of current eating patterns, preferences, and metabolic goals. The diabetes plate method is commonly used for providing basic meal planning guidance. See the full 2018 Standards of Care for a complete discussion.

\section{Weight Management}

Management and reduction of weight is important for overweight and obese people with type 1 and type 2 diabetes. Lifestyle intervention programs should be intensive and have frequent follow-up to achieve significant reductions in excess body weight and improve clinical indicators. There is strong and consistent evidence that modest persistent weight loss can delay the progression from prediabetes to type 2 diabetes and is beneficial to the management of type 2 diabetes (see Obesity Management for the Treatment of Type 2 Diabetes section).

See the full 2018 Standards of Care regarding specific recommendations for carbohydrates, protein, fats, sodium, micronutrients, and supplements. 
TABLE 4. Components of the Comprehensive Diabetes Medical Evaluation at Initial and Follow-Up Visits

\begin{tabular}{|c|c|c|c|c|}
\hline & & $\begin{array}{l}\text { INITIAL } \\
\text { VISIT }\end{array}$ & $\begin{array}{l}\text { EVERY } \\
\text { FOLLOW- } \\
\text { UP VISIT }\end{array}$ & $\begin{array}{l}\text { ANNUAL } \\
\text { VISIT }\end{array}$ \\
\hline \multirow{4}{*}{$\begin{array}{l}\text { PAST } \\
\text { MEDICAL } \\
\text { AND FAMILY } \\
\text { HISTORY }\end{array}$} & $\begin{array}{l}\text { Diabetes history } \\
\text { - Characteristics at onset (e.g., age, symptoms) } \\
\text { - Review of previous treatment regimens and response } \\
\text { - Assess frequency/cause/severity of past hospitalizations }\end{array}$ & $\begin{array}{l}\checkmark \\
\checkmark \\
\checkmark\end{array}$ & & \\
\hline & $\begin{array}{l}\text { Family history } \\
\text { - Family history of diabetes in a first-degree relative } \\
\text { - Family history of autoimmune disorder }\end{array}$ & $\begin{array}{l}\checkmark \\
\checkmark\end{array}$ & & \\
\hline & $\begin{array}{l}\text { Personal history of complications and common comorbidities } \\
\text { - Macrovascular and microvascular } \\
\text { - Common comorbidities } \\
\text { - Presence of hemoglobinopathies or anemias } \\
\text { - High blood pressure or abnormal lipids } \\
\text { - Last dental visit } \\
\text { - Last dilated eye exam } \\
\text { - Visits to specialists }\end{array}$ & $\begin{array}{l}\checkmark \\
\checkmark \\
\checkmark \\
\checkmark \\
\checkmark \\
\checkmark \\
\checkmark\end{array}$ & $\checkmark$ & $\begin{array}{l}\checkmark \\
\checkmark \\
\checkmark\end{array}$ \\
\hline & $\begin{array}{l}\text { Interval history } \\
\text { - Changes in medical/family history since last visit }\end{array}$ & & $\checkmark$ & $\checkmark$ \\
\hline \multirow[t]{2}{*}{$\begin{array}{l}\text { SOCIAL } \\
\text { HISTORY }\end{array}$} & $\begin{array}{l}\text { Assess lifestyle and behavior patterns } \\
\text { - Eating patterns and weight history } \\
\text { - Sleep behaviors and physical activity } \\
\text { - Familiarity with carbohydrate counting in type } 1 \text { diabetes } \\
\text { - Tobacco, alcohol, and substance use } \\
\text { - Identify existing social supports }\end{array}$ & $\begin{array}{l}\checkmark \\
\checkmark \\
\checkmark \\
\checkmark \\
\checkmark\end{array}$ & $\begin{array}{l}\checkmark \\
\checkmark\end{array}$ & $\begin{array}{l}\checkmark \\
\checkmark\end{array}$ \\
\hline & $\begin{array}{l}\text { Interval history } \\
\text { - Changes in social history since last visit }\end{array}$ & & $\checkmark$ & $\checkmark$ \\
\hline $\begin{array}{l}\text { MEDICATIONS } \\
\text { AND } \\
\text { VACCINATIONS }\end{array}$ & $\begin{array}{l}\text { - Medication-taking behavior } \\
\text { - Medication intolerance or side effects } \\
\text { - Complementary and alternative medicine use } \\
\text { - Vaccination history and needs }\end{array}$ & $\begin{array}{l}\checkmark \\
\checkmark \\
\checkmark \\
\checkmark\end{array}$ & $\begin{array}{l}\checkmark \\
\checkmark \\
\checkmark\end{array}$ & $\begin{array}{l}\checkmark \\
\checkmark \\
\checkmark \\
\checkmark\end{array}$ \\
\hline $\begin{array}{l}\text { TECHNOLOGY } \\
\text { USE }\end{array}$ & $\begin{array}{l}\text { - Assess use of health apps, online education, patient portals, etc. } \\
\text { - Glucose monitoring (meter/CGM): results and data use } \\
\text { - Review insulin pump settings }\end{array}$ & $\begin{array}{l}\checkmark \\
\checkmark \\
\checkmark\end{array}$ & $\begin{array}{l}\checkmark \\
\checkmark\end{array}$ & $\begin{array}{l}\checkmark \\
\checkmark \\
\checkmark\end{array}$ \\
\hline \multirow{4}{*}{ SCREENING } & $\begin{array}{l}\text { Psychosocial conditions } \\
\text { - Screen for depression, anxiety, and disordered eating; refer } \\
\text { for further assessment or intervention if warranted } \\
\text { - Consider assessment for cognitive impairment* }\end{array}$ & $\checkmark$ & & $\begin{array}{l}\checkmark \\
\checkmark\end{array}$ \\
\hline & $\begin{array}{l}\text { Diabetes self-management education and support } \\
\text { - History of dietitian/diabetes educator visits } \\
\text { - Screen for barriers to diabetes self-management } \\
\text { - Refer or offer local resources and support as needed }\end{array}$ & $\begin{array}{l}\checkmark \\
\checkmark \\
\checkmark\end{array}$ & $\checkmark$ & $\begin{array}{l}\checkmark \\
\checkmark \\
\checkmark\end{array}$ \\
\hline & $\begin{array}{l}\text { Hypoglycemia } \\
\text { - Timing of episodes, awareness, frequency and causes }\end{array}$ & $\checkmark$ & $\checkmark$ & $\checkmark$ \\
\hline & $\begin{array}{l}\text { Pregnancy planning } \\
\text { - For women with childbearing capacity, review contraceptive needs } \\
\text { and preconception planning }\end{array}$ & $\checkmark$ & $\checkmark$ & $\checkmark$ \\
\hline
\end{tabular}


TABLE 4. Components of the Comprehensive Diabetes Medical Evaluation at Initial and Follow-Up Visits, continued from p. 18

\begin{tabular}{|c|c|c|c|c|}
\hline & & $\begin{array}{l}\text { INITIAL } \\
\text { VISIT }\end{array}$ & $\begin{array}{l}\text { EVERY } \\
\text { FOLLOW- } \\
\text { UP VISIT }\end{array}$ & $\begin{array}{l}\text { ANNUAL } \\
\text { VISIT }\end{array}$ \\
\hline $\begin{array}{c}\text { PHYSICAL } \\
\text { EXAMINATION }\end{array}$ & $\begin{array}{l}\text { - Height, weight, and BMI; growth/pubertal development in children } \\
\text { and adolescents } \\
\text { - Blood pressure determination } \\
\text { - Orthostatic blood pressure measures (when indicated) } \\
\text { - Fundoscopic examination (refer to eye specialist) } \\
\text { - Thyroid palpation } \\
\text { - Skin examination (e.g., acanthosis nigricans, insulin injection or } \\
\text { insertion sites, lipodystrophy) } \\
\text { - Comprehensive foot examination } \\
\text { - Visual inspection (e.g., skin integrity, callous formation, foot } \\
\text { deformity or ulcer, toenails) } \\
\text { - Screen for PAD (pedal pulses; refer for ABI if diminished) } \\
\text { - Determination of temperature, vibration or pinprick sensation, } \\
\text { and } 10-g \text { monofilament exam }\end{array}$ & $\begin{array}{l}\checkmark \\
\checkmark \\
\checkmark \\
\checkmark \\
\checkmark \\
\checkmark \\
\checkmark \\
\checkmark \\
\checkmark\end{array}$ & $\checkmark$ & $\begin{array}{l}\checkmark \\
\checkmark \\
\checkmark \\
\checkmark \\
\checkmark \\
\checkmark \\
\checkmark \\
\checkmark\end{array}$ \\
\hline $\begin{array}{l}\text { LABORATORY } \\
\text { EVALUATION }\end{array}$ & $\begin{array}{l}\text { - A1C, if the results are not available within the past } 3 \text { months } \\
\text { - If not performed/available within the past year } \\
\text { - Lipid profile, including total, } L D L \text {, and HDL cholesterol and } \\
\text { triglycerides } \# \\
\text { - Liver function tests } \# \\
\text { - Spot urinary albumin-to-creatinine ratio } \\
\text { - Serum creatinine and estimated glomerular filtration rate }{ }^{\dagger} \\
\text { - Thyroid-stimulating hormone in patients with type } 1 \text { diabetes } \# \\
\text { - Vitamin B12 if on metformin (when indicated) } \\
\text { - Serum potassium levels in patients on ACE inhibitors, ARBs, or } \\
\text { diuretics }{ }^{\dagger}\end{array}$ & $\begin{array}{l}\checkmark \\
\checkmark \\
\checkmark \\
\checkmark \\
\checkmark \\
\checkmark \\
\checkmark \\
\checkmark\end{array}$ & $\checkmark$ & $\begin{array}{l}\checkmark \\
\checkmark^{\wedge} \\
\checkmark \\
\checkmark \\
\checkmark \\
\checkmark \\
\checkmark\end{array}$ \\
\hline \multirow{3}{*}{$\begin{array}{c}\text { ASSESSMENT } \\
\text { AND PLAN }\end{array}$} & $\begin{array}{l}\text { Goal setting } \\
\text { - Set } \mathrm{A} 1 \mathrm{C} / \text { blood glucose target and monitoring frequency } \\
\text { - If hypertension diagnosed, establish blood pressure goal } \\
\text { - Incorporate new members to the care team as needed } \\
\text { - Diabetes education and self-management support needs }\end{array}$ & $\begin{array}{l}\checkmark \\
\checkmark \\
\checkmark \\
\checkmark\end{array}$ & $\begin{array}{l}\checkmark \\
\checkmark \\
\checkmark\end{array}$ & $\begin{array}{l}\checkmark \\
\checkmark \\
\checkmark \\
\checkmark\end{array}$ \\
\hline & $\begin{array}{l}\text { Cardiovascular risk assessment and staging of CKD } \\
\text { - History of ASCVD } \\
\text { - Presence of ASCVD risk factors (see Table 9.2) } \\
\text { - Staging of CKD (see Table 10.1) }\end{array}$ & $\begin{array}{l}\checkmark \\
\checkmark \\
\checkmark\end{array}$ & $\begin{array}{l}\checkmark \\
\checkmark \\
\checkmark\end{array}$ & $\begin{array}{l}\checkmark \\
\checkmark \\
\checkmark\end{array}$ \\
\hline & $\begin{array}{l}\text { Therapeutic treatment plan } \\
\text { - Lifestyle management } \\
\text { - Pharmacologic therapy } \\
\text { - Referrals to specialists (including dietitian and diabetes educator) } \\
\text { as needed } \\
\text { - Use of glucose monitoring and insulin delivery devices }\end{array}$ & $\begin{array}{l}\checkmark \\
\checkmark \\
\checkmark \\
\checkmark\end{array}$ & $\begin{array}{l}\checkmark \\
\checkmark \\
\checkmark \\
\checkmark\end{array}$ & $\begin{array}{l}\checkmark \\
\checkmark \\
\checkmark \\
\checkmark\end{array}$ \\
\hline
\end{tabular}

Tables 9.2 and 10. 1 are in the full 2018 Standards of Care. ${ }^{\star} \geq 65$ years. +May be needed more frequently in patients with known CKD or with changes in medications that affect kidney function and serum potassium (see Table 10.2 in the full Standards of Care). \#May also need to be checked after initiation or dose changes of medications that affect these laboratory values (i.e., diabetes, blood pressure, cholesterol, or thyroid medications). $\wedge$ In people without dyslipidemia and not on cholesterol-lowering therapy, testing may be less frequent. $A B I$, ankle-brachial pressure index. 
Routine supplementation with antioxidants, such as vitamins $\mathrm{E}$ and $\mathrm{C}$ and carotene, is not advised due to lack of evidence of efficacy and concern related to long-term safety. In addition, there is insufficient evidence to support the routine use of herbals and micronutrients, such as cinnamon and vitamin $\mathrm{D}$, to improve glycemic control in people with diabetes.

\section{Alcohol}

Moderate alcohol intake does not have major detrimental effects on long-term blood glucose control in people with diabetes. Risks associated with alcohol consumption include hypoglycemia (particularly for those using insulin or insulin secretagogue therapies), weight gain, and hyperglycemia (for those consuming excessive amounts). People with diabetes can follow the same guidelines as those without diabetes if they choose to drink.

\section{Nonnutritive Sweeteners}

For some people with diabetes who are accustomed to sugar-sweetened products, nonnutritive sweeteners (containing few or no calories) may be an acceptable substitute for nutritive sweeteners. Most systematic reviews and meta-analyses show benefits for nonnutritive sweetener use in weight loss; however, some studies suggest an association with weight gain.

\section{Physical Activity}

\section{Recommendations}

- Children and adolescents with type 1 or type 2 diabetes or prediabetes should engage in $60 \mathrm{~min} /$ day or more of moderate- or vigorousintensity aerobic activity, with vigorous muscle-strengthening and bone-strengthening activities at least 3 days/week. C

- Most adults with type $1 \mathrm{C}$ and type $2 \mathrm{~B}$ diabetes should engage in $150 \mathrm{~min}$ or more of moderateto-vigorous intensity aerobic activity per week, spread over at least 3 days/week, with no more than 2 consecutive days without activity. Shorter durations (minimum 75 $\mathrm{min} /$ week) of vigorous-intensity or interval training may be sufficient for younger and more physically fit individuals.

- Adults with type $1 \mathrm{C}$ and type 2 B diabetes should engage in 2-3 sessions/week of resistance exercise on nonconsecutive days.

- All adults, and particularly those with type 2 diabetes, should decrease the amount of time spent in daily sedentary behavior. B Prolonged sitting should be interrupted every $30 \mathrm{~min}$ for blood glucose benefits, particularly in adults with type 2 diabetes. $C$

- Flexibility training and balance training are recommended 2-3 times/week for older adults with diabetes. Yoga and tai chi may be included based on individual preferences to increase flexibility, muscular strength, and balance. $C$

\section{Exercise in the Presence of Specific Long-term Complications of Diabetes}

Retinopathy

If proliferative diabetic retinopathy or severe nonproliferative diabetic retinopathy is present, then vigorousintensity aerobic or resistance exercise may be contraindicated because of the risk of triggering vitreous hemorrhage or retinal detachment. Consultation with an ophthalmologist prior to engaging in an intense exercise regimen may be appropriate.

\section{Peripheral Neuropathy}

Decreased pain sensation and a higher pain threshold in the extremities result in an increased risk of skin breakdown, infection, and Charcot joint destruction with some forms of exercise.

\section{Autonomic Neuropathy}

Autonomic neuropathy can increase the risk of exercise-induced injury or adverse events through decreased cardiac responsiveness to exercise, postural hypotension, impaired thermoregulation, impaired night vision due to impaired papillary reaction, and greater susceptibility to hypoglycemia. Cardiovascular autonomic neu- ropathy is also an independent risk factor for cardiovascular death and silent myocardial ischemia. Therefore, individuals with diabetic autonomic neuropathy should undergo cardiac investigation before beginning physical activity more intense than that to which they are accustomed.

\section{Psychosocial Issues}

\section{Recommendations}

- Psychosocial care should be integrated with a collaborative, patientcentered approach and provided to all people with diabetes, with the goals of optimizing health outcomes and health-related quality of life. A

- Psychosocial screening and followup may include, but are not limited to, attitudes about diabetes, expectations for medical management and outcomes, affect or mood, general and diabetes-related quality of life, available resources (financial, social, and emotional), and psychiatric history. E

- Providers should consider assessment for symptoms of diabetes distress, depression, anxiety, disordered eating, and cognitive capacities using patient-appropriate standardized and validated tools at the initial visit, at periodic intervals, and when there is a change in disease, treatment, or life circumstance. Including caregivers and family members in this assessment is recommended. $\mathrm{B}$

- Consider screening older adults (aged $\geq 65$ years) with diabetes for cognitive impairment and depression. $\mathbf{B}$

\section{Diabetes Distress}

\section{Recommendation}

- Routinely monitor people with diabetes for diabetes distress (DD), particularly when treatment targets are not met and/or at the onset of diabetes complications. B

DD is very common and is distinct from other psychological disorders. 
DD refers to significant negative psychological reactions related to emotional burdens and worries specific to an individual's experience in having to manage a severe, complicated, and demanding chronic disease such as diabetes.

DD should be routinely monitored using patient-appropriate validated measures. If DD is identified, the person should be referred for specific diabetes education to address areas of diabetes self-care that are most relevant to the patient and impact clinical management.

\section{Serious Mental Illness}

\section{Recommendations}

- Annually screen people who are prescribed atypical antipsychotic medications for prediabetes or diabetes. B

- If a second-generation antipsychotic medication is prescribed for adolescents or adults with diabetes, changes in weight, glycemic control, and cholesterol levels should be carefully monitored and the treatment regimen should be reassessed. C

- Incorporate monitoring of diabetes self-care activities into treatment goals in people with diabetes and serious mental illness. B

Table 5 lists considerations regarding referral to a mental health specialist. Refer to the ADA position statement "Psychosocial Care for People With Diabetes" for a list of assessment tools and additional details.

\section{PREVENTION OR DELAY OF TYPE 2 DIABETES}

\section{Recommendations}

- At least annual monitoring for the development of diabetes in those with prediabetes is suggested. E

- Patients with prediabetes should be referred to an intensive behavioral lifestyle intervention program modeled on the Diabetes Prevention Program to achieve and maintain $7 \%$ loss of initial body weight and increase

TABLE 5. Situations That Warrant Referral of a Person With Diabetes to a Mental Health Provider for Evaluation and Treatment

- If self-care remains impaired in a person with DD after tailored diabetes education

- If a person has a positive screen on a validated screening tool for depressive symptoms

- In the presence of symptoms or suspicions of disordered eating behavior, an eating disorder, or disrupted patterns of eating

- If intentional omission of insulin or oral medication to cause weight loss is identified

- If a person has a positive screen for anxiety or fear of hypoglycemia

- If a serious mental illness is suspected

- In youth and families with behavioral self-care difficulties, repeated hospitalizations for diabetic ketoacidosis, or significant distress

- If a person screens positive for cognitive impairment

- Declining or impaired ability to perform diabetes self-care behaviors

- Before undergoing bariatric or metabolic surgery and after surgery if assessment reveals an ongoing need for adjustment support

moderate-intensity physical activity (such as brisk walking) to at least $150 \mathrm{~min} /$ week. A

\section{Pharmacologic Interventions}

\section{Recommendations}

- Metformin therapy for prevention of type 2 diabetes should be considered in those with prediabetes, especially for those with BMI $\geq 35$ $\mathrm{kg} / \mathrm{m}^{2}$, those aged $<60$ years, and women with prior gestational diabetes mellitus. A

- Long-term use of metformin may be associated with biochemical vitamin B12 deficiency, and periodic measurement of vitamin B12 levels should be considered in metformin-treated patients, especially in those with anemia or peripheral neuropathy. $B$

Pharmacologic agents including metformin, $\alpha$-glucosidase inhibitors, orlistat, glucagon-like peptide 1 (GLP-1) receptor agonists, and thiazolidinediones have each been shown to decrease incident diabetes to various degrees in those with prediabetes in research studies, though none are approved by the U.S. Food and Drug Administration specifically for diabetes prevention. One has to balance the risk/benefit of each medication.
Metformin has the strongest evidence base and demonstrated long-term safety as pharmacologic therapy for diabetes prevention. For other drugs, cost, side effects, and durable efficacy require consideration.

\section{GLYCEMIC TARGETS}

\section{Assessment of Glycemic Control}

Self-monitoring of blood glucose (SMBG) frequency and timing should be dictated by patients' specific needs and goals. SMBG is especially important for patients treated with insulin to monitor for and prevent asymptomatic hypoglycemia and hyperglycemia. Data indicate similar A1C and safety with the use of continuous glucose monitoring (CGM) compared with SMBG.

\section{Recommendations}

- Most patients using intensive insulin regimens (a multiple daily injection [MDI] regimen or insulin pump therapy) should perform SMBG prior to meals and snacks, at bedtime, occasionally postprandially, prior to exercise, when they suspect low blood glucose, after treating low blood glucose until they are normoglycemic, 
and prior to critical tasks such as driving. $B$

SMBG allows patients to evaluate their responses to therapy and assess whether glycemic targets are being met. It can help in preventing hypoglycemia and adjusting medications (particularly prandial insulin), MNT, and physical activity. Evidence supports a correlation between SMBG frequency and lower A1C in insulintreated patients. However, evidence is lacking on when and how often SMBG is needed for patients who do not use intensive insulin regimens such as those with type 2 diabetes using oral agents and/or basal insulin. Randomized trials have called into question the clinical utility and cost-effectiveness of routine SMBG in noninsulin-treated patients.

SMBG accuracy is dependent on both the instrument and the user. Evaluate each patient's technique initially and at regular intervals thereafter. Evaluate the ongoing need for and frequency of SMBG at each visit. A guide to $S M B G$ products can be found at forecast.diabetes.org/ consumerguide.

CGM measures interstitial glucose (which correlates well with plasma glucose), and most CGM devices include alarms for both hypoglycemic and hyperglycemic excursions. A "flash" CGM device, which does not have alarm functions, was recently approved for use in adults.

\section{A1C Testing}

\section{Recommendations}

- Perform the A1C test at least two times a year in patients who are meeting treatment goals (and who have stable glycemic control). E

- Perform the A1C test quarterly in patients whose therapy has changed or who are not meeting glycemic goals. E

- Point-of-care (POC) testing for A1C provides the opportunity for more timely treatment changes. E

TABLE 6. Summary of Glycemic Recommendations for Many Nonpregnant Adults With Diabetes

\begin{tabular}{lc}
\hline A1C & $<7.0 \%(53 \mathrm{mmol} / \mathrm{mol})^{\star}$ \\
\hline Preprandial capillary plasma glucose & $80-130 \mathrm{mg} / \mathrm{dL}(4.4-7.2 \mathrm{mmol} / \mathrm{L})^{\star}$ \\
\hline $\begin{array}{l}\text { Peak postprandial capillary plasma } \\
\text { glucoset }\end{array}$ & $<180 \mathrm{mg} / \mathrm{dL}(10.0 \mathrm{mmol} / \mathrm{L})^{\star}$ \\
\hline
\end{tabular}

*More or less stringent glycemic goals may be appropriate for individual patients. Goals should be individualized based on duration of diabetes, age/life expectancy, comorbid conditions, known CVD or advanced microvascular complications, hypoglycemia unawareness, and individual patient considerations. TPostprandial glucose may be targeted if A1C goals are not met despite reaching preprandial glucose goals. Postprandial glucose measurements should be made 1-2 h after the beginning of the meal, generally peak levels in patients with diabetes.

\section{A1C Goals}

\section{Recommendations}

- A reasonable A1C goal for many nonpregnant adults is $<7 \%(53$ $\mathrm{mmol} / \mathrm{mol}$ ). A

- Providers might reasonably suggest more stringent $\mathrm{A} 1 \mathrm{C}$ goals (such as $<6.5 \%[48 \mathrm{mmol} / \mathrm{mol}]$ ) for selected individual patients if this can be achieved without significant hypoglycemia or other adverse effects of treatment (i.e., polypharmacy). Appropriate patients might include those with short duration of diabetes, type 2 diabetes treated with lifestyle or metformin only, long life expectancy, or no significant CVD. C

- Less stringent A1C goals (such as $<8 \%[64 \mathrm{mmol} / \mathrm{mol}]$ ) may be appropriate for patients with a history of severe hypoglycemia, limited life expectancy, advanced microvascular or macrovascular complications, extensive comorbid conditions, or long-standing diabetes in whom the goal is difficult to achieve despite diabetes self-management education, appropriate glucose monitoring, and effective doses of multiple glucose-lowering agents including insulin. B

The full 2018 Standards of Care provide the justification for current glycemic control recommendations. See Children and Adolescents and Management of Diabetes in Pregnancy sections for A1C goals for these populations. Table 6 summarizes glycemic recommendations for many nonpregnant adults. Figure 1 depicts factors used to determine $\mathrm{A} 1 \mathrm{C}$ targets for individual patients.

\section{Hypoglycemia}

\section{Recommendations}

- Individuals at risk for hypoglycemia should be asked about symptomatic and asymptomatic hypoglycemia at each encounter. C

- Glucose (15-20 g) is the preferred treatment for the conscious individual with blood glucose $\leq 70$ $\mathrm{mg} / \mathrm{dL}[3.9 \mathrm{mmol} / \mathrm{L}]$ ), although any form of carbohydrate that contains glucose may be used. Fifteen minutes after treatment, if SMBG shows continued hypoglycemia, the treatment should be repeated. Once SMBG returns to normal, the individual should consume a meal or snack to prevent recurrence of hypoglycemia. E

- Glucagon should be prescribed for all individuals at increased risk of clinically significant hypoglycemia, defined as blood glucose $<54 \mathrm{mg} / \mathrm{dL}(3.0 \mathrm{mmol} / \mathrm{L})$, so it is available should it be needed. Caregivers, school personnel, or family members of these individuals should know where it is and when and how to administer it. Glucagon administration is not limited to health care professionals. E 
Approach to the Management of Hyperglycemia

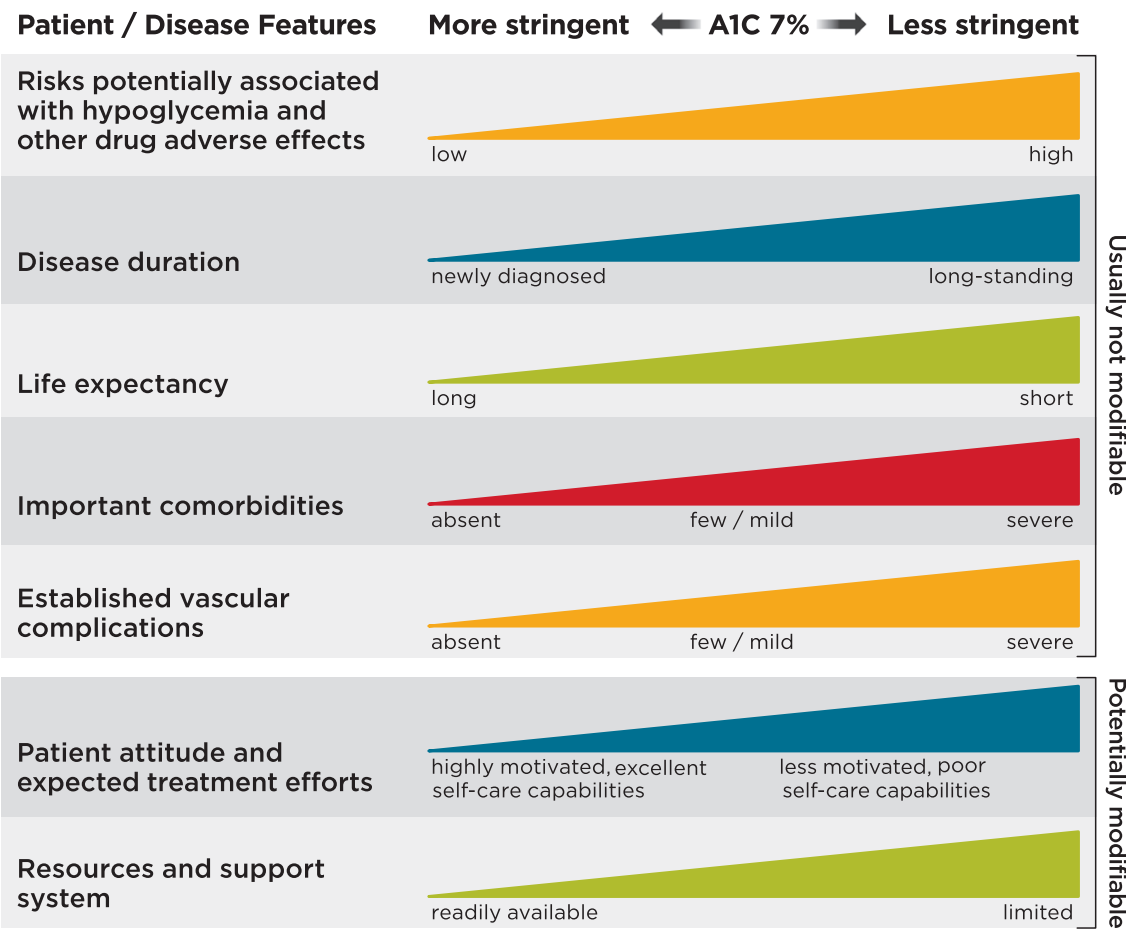

FIGURE 1. Depicted are patient and disease factors used to determine optimal A1C targets. Characteristics and predicaments toward the left justify more stringent efforts to lower A1C; those toward the right suggest less stringent efforts. Adapted with permission from Inzucchi et al. Diabetes Care 2015;38:140-149.

- Hypoglycemia unawareness or one or more episodes of severe hypoglycemia should trigger reevaluation of the treatment regimen. $E$

- Insulin-treated patients with hypoglycemia unawareness or an episode of clinically significant hypoglycemia should be advised to raise their glycemic targets to strictly avoid hypoglycemia for at least several weeks in order to partially reverse hypoglycemia unawareness and reduce risk of future episodes. A

- Ongoing assessment of cognitive function is suggested with increased vigilance for hypoglycemia by the clinician, patient, and caregivers if low cognition or declining cognition is found. $B$

\section{OBESITY MANAGEMENT FOR THE TREATMENT OF TYPE 2 DIABETES}

There is strong and consistent evidence that obesity management can delay the progression from prediabetes to type 2 diabetes and may be beneficial in the treatment of type 2 diabetes. In overweight and obese patients with type 2 diabetes, modest and sustained weight loss has been shown to improve glycemic control and to reduce the need for glucoselowering medications.

\section{Recommendation}

- At each patient encounter, BMI should be calculated and documented in the medical record. $\mathrm{B}$

Providers should advise overweight and obese patients that, in general, higher BMIs increase the risk of CVD and all-cause mortality. Providers should assess each patient's readiness to achieve weight loss and jointly determine weight loss goals and intervention strategies.

\section{Diet, Physical Activity, and Behavioral Therapy}

\section{Recommendations}

- Diet, physical activity, and behavioral therapy designed to achieve
$>5 \%$ weight loss should be prescribed for overweight and obese patients with type 2 diabetes ready to achieve weight loss. A

- Such interventions should be high intensity ( $\geq 16$ sessions in 6 months) and focus on diet, physical activity, and behavioral strategies to achieve a 500-750 $\mathrm{kcal} /$ day energy deficit. A

- Diets should be individualized, as those that provide the same caloric restriction but differ in protein, carbohydrate, and fat content are equally effective in achieving weight loss. A

- For patients who achieve shortterm weight-loss goals, long-term $(\geq 1$ year) comprehensive weight maintenance programs should be prescribed. Such programs should provide at least monthly contact and encourage ongoing monitoring of body weight (weekly or more frequently), continued consumption of a reduced-calorie diet, and participation in high levels of physical activity (200-300 $\mathrm{min}$ (week). A

\section{Pharmacotherapy}

\section{Recommendations}

- When choosing glucose-lowering medications for overweight or obese patients with type 2 diabetes, consider their effect on weight. $\mathrm{E}$

- Whenever possible, minimize the medications for comorbid conditions that are associated with weight gain. $\mathrm{E}$

- Weight loss medications may be effective as adjuncts to diet, physical activity, and behavioral counseling for selected patients with type 2 diabetes and $\mathrm{BMI} \geq 27$ $\mathrm{kg} / \mathrm{m}^{2}$. Potential benefits must be weighed against the potential risks of the medications. A

- If a patient's response to weight loss medications is $<5 \%$ weight loss after 3 months or if there are any safety or tolerability issues at any time, the medication should be discontinued and alternative med- 
ications or treatment approaches should be considered. A

\section{Metabolic Surgery}

\section{Recommendations}

- Metabolic surgery should be recommended as an option to treat type 2 diabetes in appropriate surgical candidates with $\mathrm{BMI} \geq 40$ $\mathrm{kg} / \mathrm{m}^{2}$ (BMI $\geq 37.5 \mathrm{~kg} / \mathrm{m}^{2}$ in Asian Americans), regardless of the level of glycemic control or complexity of glucose-lowering regimens, and in adults with BMI 35.0-39.9 $\mathrm{kg} / \mathrm{m}^{2}\left(32.5-37.4 \mathrm{~kg} / \mathrm{m}^{2}\right.$ in Asian Americans) when hyperglycemia is inadequately controlled despite lifestyle and optimal medical therapy. A

- Metabolic surgery should be considered as an option for adults with type 2 diabetes and BMI $30.0-34.9 \quad \mathrm{~kg} / \mathrm{m}^{2} \quad(27.5-32.4$ $\mathrm{kg} / \mathrm{m}^{2}$ in Asian Americans) if hyperglycemia is inadequately controlled despite optimal medical control by either oral or injectable medications (including insulin). B

- Metabolic surgery should be performed in high-volume centers with multidisciplinary teams that understand and are experienced in the management of diabetes and gastrointestinal surgery. C

- Long-term lifestyle support and routine monitoring of micronutrient and nutritional status must be provided to patients after surgery, according to guidelines for postoperative management of metabolic surgery by national and international professional societies. C

- People presenting for metabolic surgery should receive a comprehensive mental health assessment. B Surgery should be postponed in patients with histories of alcohol or substance abuse, significant depression, suicidal ideation, or other mental health conditions until these conditions have been fully addressed. E

- People who undergo metabolic surgery should be evaluated to assess the need for ongoing men- tal health services to help them adjust to medical and psychosocial changes after surgery. $C$

A substantial body of evidence has now accumulated, including data from numerous randomized controlled clinical trials, regarding benefit of metabolic surgery in appropriate patients.

\section{PHARMACOLOGIC APPROACHES TO GLYCEMIC TREATMENT}

\section{Pharmacologic Therapy for Type 1 Diabetes}

\section{Recommendations}

- Most people with type 1 diabetes should be treated with an MDI regimen of prandial insulin and basal insulin or continuous subcutaneous insulin infusion (CSII). A

- Most individuals with type 1 diabetes should use rapid-acting insulin analogs to reduce hypoglycemia risk. A

- Consider educating individuals with type 1 diabetes on matching prandial insulin doses to carbohydrate intake, premeal blood glucose levels, and anticipated physical activity. E

- Individuals with type 1 diabetes who have been successfully using CSII should have continued access to this therapy after they turn 65 years of age. E

\section{Pharmacologic Therapy for Type 2 Diabetes}

\section{Recommendations}

- Metformin, if not contraindicated and if tolerated, is the preferred initial pharmacologic agent for the treatment of type 2 diabetes. A

- Consider initiating insulin therapy (with or without additional agents) in patients with newly diagnosed type 2 diabetes who are symptomatic and/or have $\mathrm{A} 1 \mathrm{C} \geq 10 \%$ (86 $\mathrm{mmol} / \mathrm{mol}$ ) and/or blood glucose levels $\geq 300 \mathrm{mg} / \mathrm{dL}$ (16.7 mmol/L). E

- Consider initiating dual therapy in patients with newly diagnosed type 2 diabetes who have A1C $\geq 9 \%(75 \mathrm{mmol} / \mathrm{mol})$. E

- In patients without atherosclerotic CVD (ASCVD), if monotherapy or dual therapy does not achieve or maintain the A1C goal over 3 months, add an additional antihyperglycemic agent based on drug-specific and patient factors. A

- A patient-centered approach should be used to guide the choice of pharmacologic agents. Considerations include efficacy, hypoglycemia risk, history of ASCVD, impact on weight, potential side effects, renal effects, delivery method (oral versus subcutaneous), cost, and patient preferences. $\mathrm{E}$

- In patients with type 2 diabetes and established ASCVD, antihyperglycemic therapy should begin with lifestyle management and metformin and subsequently incorporate an agent proven to reduce major adverse cardiovascular events and cardiovascular mortality (currently empagliflozin and liraglutide), after considering drug-specific and patient factors. (Table 7) A*

- In patients with type 2 diabetes and established ASCVD, after lifestyle management and metformin, the antihyperglycemic agent canagliflozin may be considered to reduce major adverse cardiovascular events, based on drug-specific and patient factors (Table 7). C*

- Continuous reevaluation of the medication regimen and adjustment as needed to incorporate patient factors (Table 7) and regimen complexity is recommended. E

- For patients with type 2 diabetes who are not achieving glycemic goals, drug intensification, including consideration of insulin therapy, should not be delayed. B

- Metformin should be continued when used in combination with other agents, including insulin, if 
not contraindicated and if tolerated. A

*See Figure 2 for more information.

Table 7 highlights considerations for a patient-centered approach to diabetes pharmacologic therapies. Figure 2 and Figure 3 outline monotherapy and combination therapy emphasizing drugs commonly used in the United States and/or Europe. See Table 8.2 in the full Standards of Care for information on the pharmacology of glucose-lowering agents available in the United States for the treatment of type 2 diabetes.

\section{CVD AND RISK MANAGEMENT}

ASCVD_defined as coronary heart disease, cerebrovascular disease, or peripheral arterial disease (PAD) presumed to be of atherosclerotic origin - is the leading cause of morbidity and mortality for individuals with diabetes and is the largest contributor to the direct and indirect costs of diabetes. Common conditions coexisting with type 2 diabetes (e.g., hypertension and dyslipidemia) are clear risk factors for ASCVD, and diabetes itself confers independent risk.

Therefore, cardiovascular risk factors should be systematically assessed at least annually in all patients with diabetes. These risk factors include hypertension, dyslipidemia, smoking, a family history of premature coronary disease, chronic kidney disease (CKD), and the presence of albuminuria.

\section{Hypertension/Blood Pressure Control}

\section{Recommendations}

- Blood pressure should be measured at every routine clinical visit. Patients found to have elevated blood pressure $(\geq 140 / 90 \mathrm{mmHg})$ should have blood pressure confirmed using multiple readings, including measurements on a separate day, to diagnose hypertension. B

- All hypertensive patients with diabetes should monitor their blood pressure at home. B
- Most patients with diabetes and hypertension should be treated to a systolic blood pressure goal of $<140 \mathrm{mmHg}$ and a diastolic blood pressure goal of $<90 \mathrm{mmHg}$. A

- Lower systolic and diastolic blood pressure targets, such as 130/80 $\mathrm{mmHg}$, may be appropriate for individuals at high risk of CVD, if they can be achieved without undue treatment burden. $C$

- For patients with blood pressure $>120 / 80 \mathrm{mmHg}$, lifestyle intervention consists of weight loss if overweight or obese; a Dietary Approaches to Stop Hypertension-style dietary pattern including reducing sodium and increasing potassium intake; moderation of alcohol intake; and increased physical activity. B

- Patients with confirmed officebased blood pressure $\geq 140 / 90$ $\mathrm{mmHg}$ should, in addition to lifestyle therapy, have prompt initiation and timely titration of pharmacologic therapy to achieve blood pressure goals. A

- Patients with confirmed officebased blood pressure $\geq 160 / 100$ $\mathrm{mmHg}$ should, in addition to lifestyle therapy, have prompt initiation and timely titration of two drugs or a single-pill combination of drugs demonstrated to reduce cardiovascular events in patients with diabetes. A

- Treatment for hypertension should include drug classes demonstrated to reduce cardiovascular events in patients with diabetes (ACE inhibitors, angiotensin receptor blockers [ARBs], thiazide-like diuretics, or dihydropyridine calcium channel blockers). A

- Multiple-drug therapy is generally required to achieve blood pressure targets. However, combinations of ACE inhibitors and ARBs and combinations of ACE inhibitors or $A R B s$ with direct renin inhibitors should not be used. A

- An ACE inhibitor or ARB, at the maximumly tolerated dose indicated for blood pressure treatment, is the recommended first-line treatment for hypertension in patients with diabetes and urinary albumin-to-creatinine ratio (UACR) $\geq 300 \mathrm{mg} / \mathrm{g}$ creatinine (Cr) A or $30-299 \mathrm{mg} / \mathrm{g}$ Cr. $B$ If one class is not tolerated, the other should be substituted. B

- For patients treated with an ACE inhibitor, $A R B$, or diuretic, serum creatinine/estimated glomerular filtration rate (eGFR) and serum potassium levels should be monitored at least annually. B

Figure 9.1 in the full 2018 Standards of Care summarizes recommendations for the treatment of confirmed hypertension in people with diabetes. Based on current evidence, ADA recommends hypertension diagnosis and treatment as outlined, emphasizing individualization of blood pressure targets. ADA is aware of hypertension recommendations from other organizations. The ADA Professional Practice Committee continuously reviews and considers all studies, particularly high-quality trials including people with diabetes, for potential incorporation in future recommendations.

\section{Lipid Management}

\section{Recommendations}

- Lifestyle modification focusing on weight loss (if indicated); the reduction of saturated fat, trans fat, and cholesterol intake; increase of dietary $\omega-3$ fatty acids, viscous fiber, and plant stanols/sterols intake; and increased physical activity should be recommended to improve the lipid profile in patients with diabetes. A

- Intensify lifestyle therapy and optimize glycemic control for patients with elevated triglyceride levels $(\geq 150 \mathrm{mg} / \mathrm{dL}[1.7 \mathrm{mmol} / \mathrm{L}])$ and $/$ or low HDL cholesterol $(<40 \mathrm{mg} / \mathrm{dL}$ $[1.0 \mathrm{mmol} / \mathrm{L}]$ for men, $<50 \mathrm{mg} / \mathrm{dL}$ [1.3 mmol/L] for women). C

- Obtain a lipid profile at initiation of statins or other lipid-lowering therapy, 4-12 weeks after ini- 


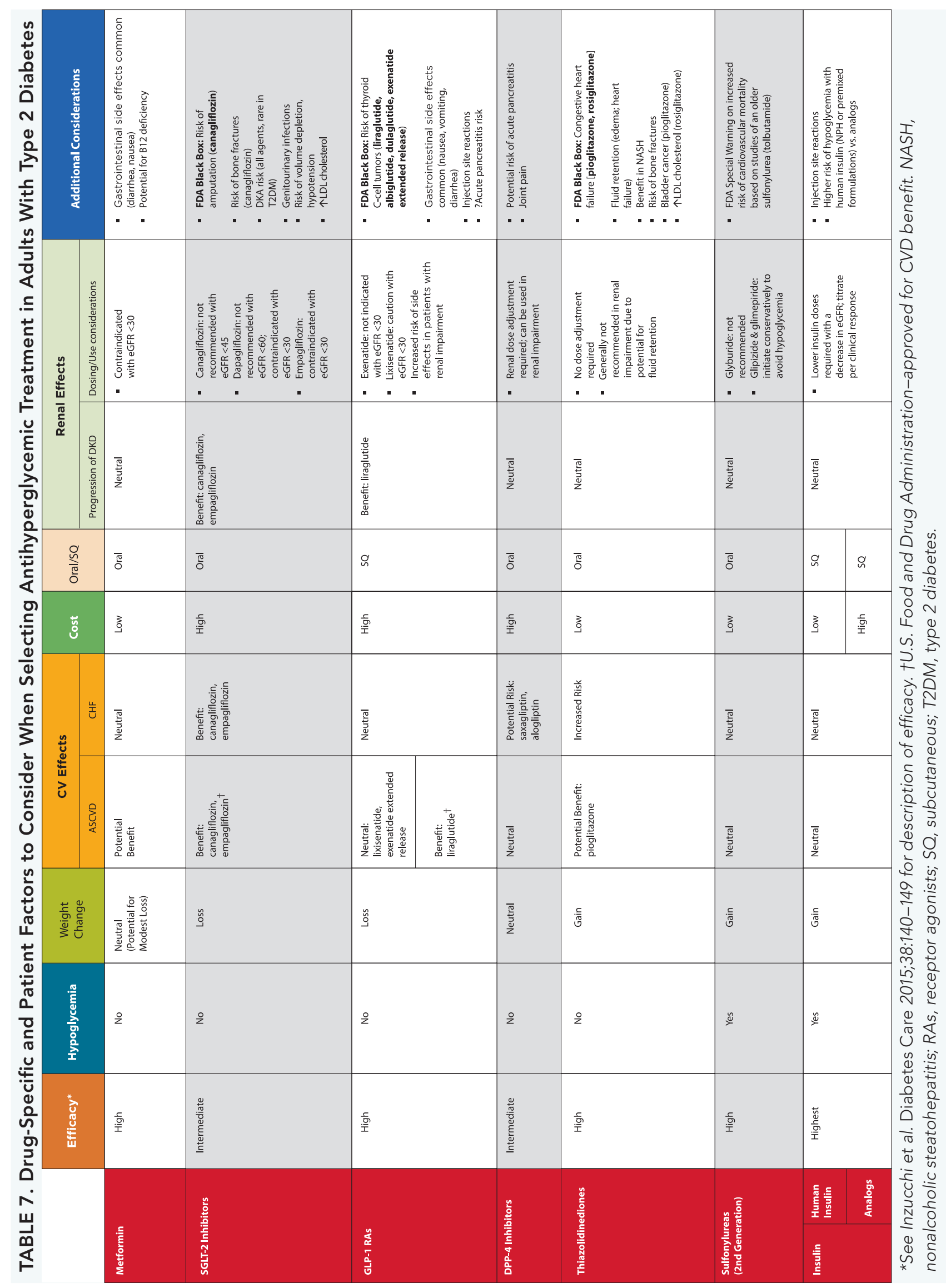




\section{Antihyperglycemic Therapy in Adults with Type 2 Diabetes}

\section{At diagnosis, initiate lifestyle management, set A1C target, and initiate pharmacologic therapy based on A1C:}

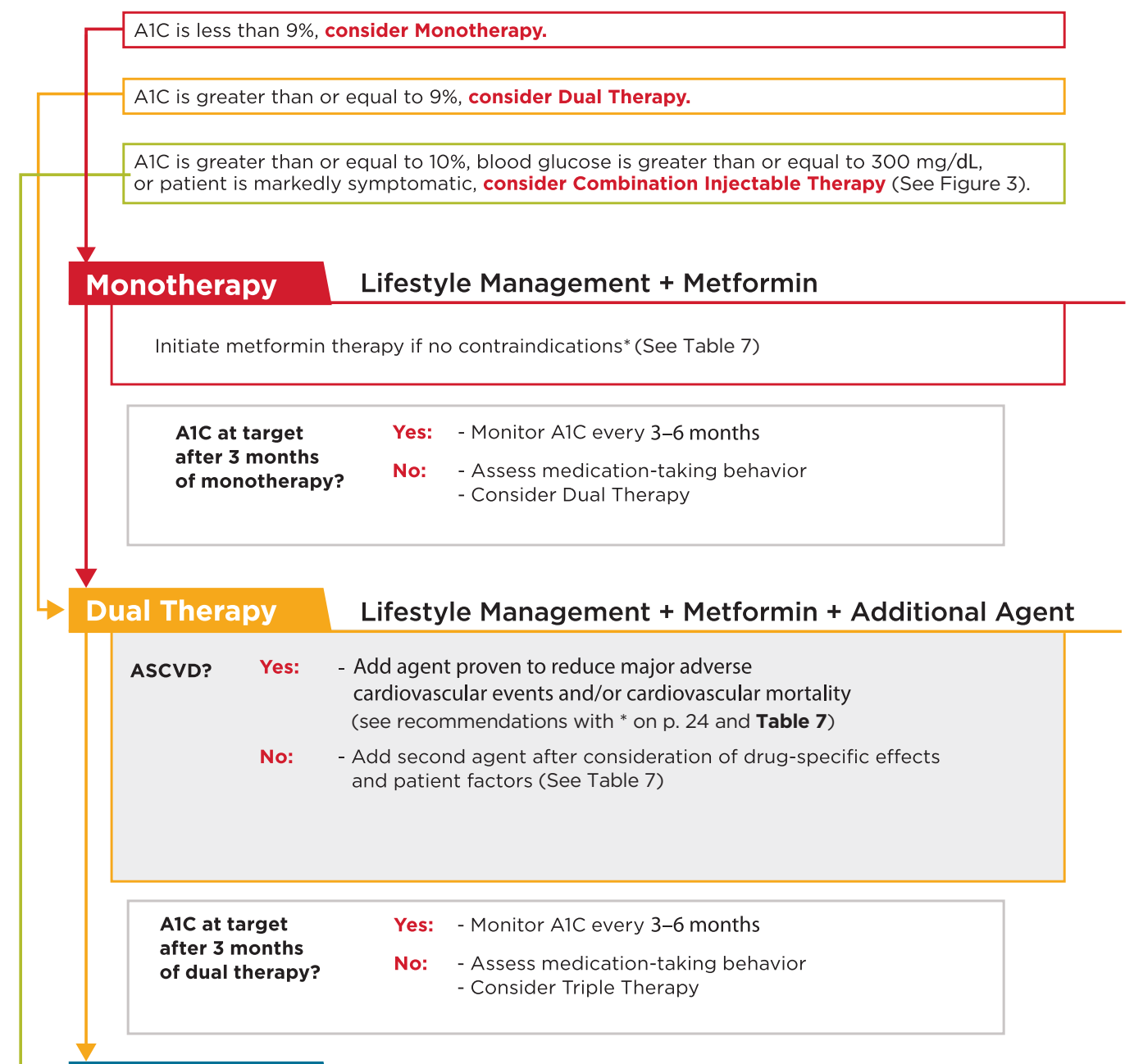

Triple Therapy Lifestyle Management + Metformin + Two Additional Agents

Add third agent based on drug-specific effects and patient factors \# (See Table 7)

$\begin{array}{lll}\begin{array}{l}\text { A1C at target } \\ \text { after } 3 \text { months } \\ \text { of triple therapy? }\end{array} & \text { Yes: } & \text { - Monitor A1C every 3-6 months } \\ & & \text { - Assess medication-taking behavior } \\ & \text { - Consider Combination Injectable Therapy (See Figure 3). }\end{array}$

FIGURE 2. Antihyperglycemic therapy in type 2 diabetes: general recommendations. *If patient does not tolerate or has contraindications to metformin, consider agents from another class in Table 7. \#GLP-1 receptor agonists and DPP-4 inhibitors should not be prescribed in combination. If a patient with ASCVD is not yet on an agent with evidence of cardiovascular risk reduction, consider adding. 
Initiate Basal Insulin

Usually with metformin +/- other noninsulin agent

Start: $10 \mathrm{U} /$ day or $0.1-0.2 \mathrm{U} / \mathrm{kg} /$ day

Adjust: $10-15 \%$ or 2-4 units once or twice weekly to reach FBG target

For hypo: Determine \& address cause; if no clear reason for hypo,

$\downarrow$ dose by 4 units or $10-20 \%$

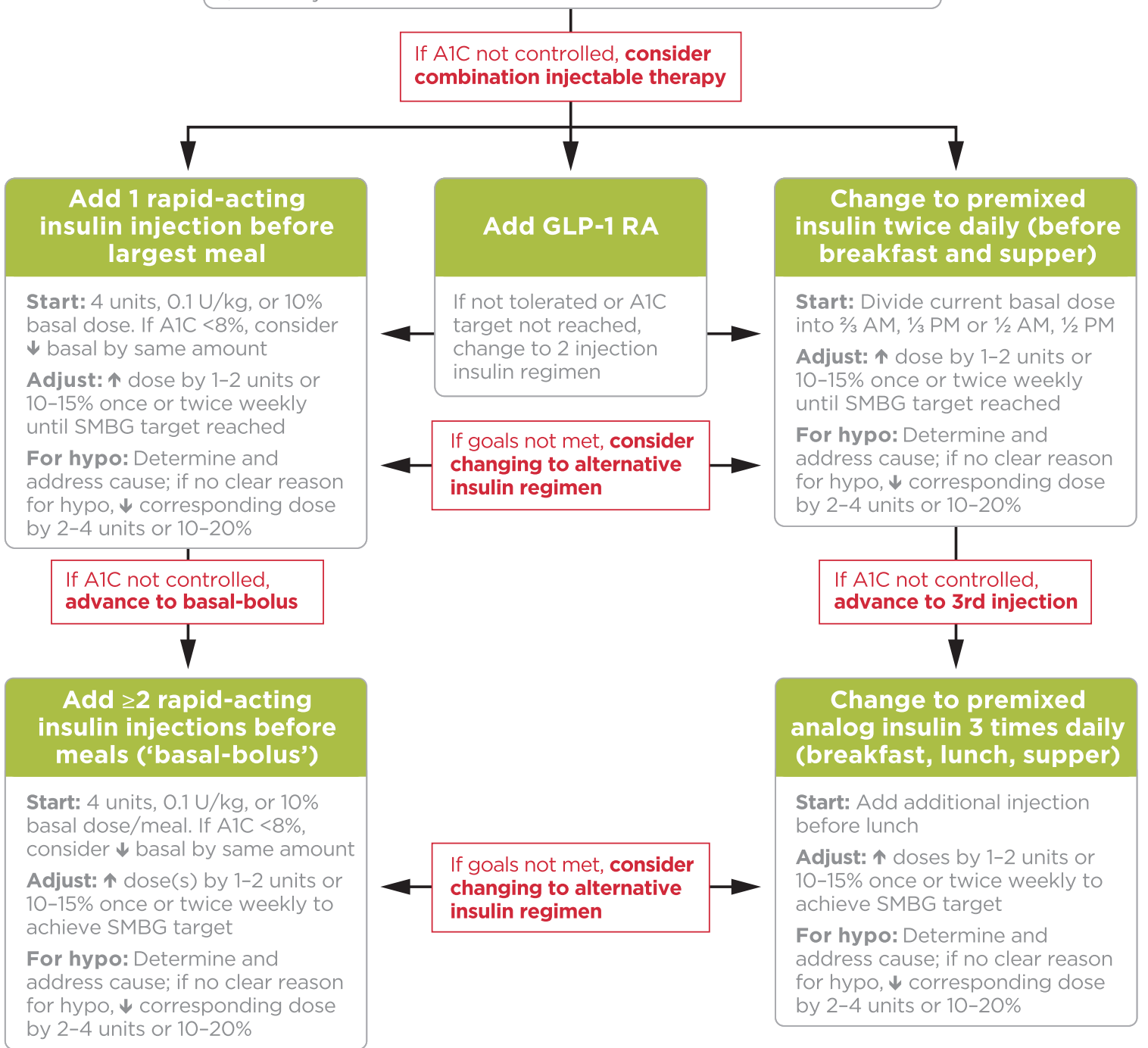

FIGURE 3. Combination injectable therapy for type 2 diabetes. FBG, fasting blood glucose; hypo, hypoglycemia. Adapted with permission from Inzucchi et al. Diabetes Care 2015;38:140-149.

tiation or a change in dose, and annually thereafter as it may help to monitor the response to therapy and inform adherence. $\mathrm{E}$

- For patients of all ages with diabetes and ASCVD, high-intensity statin therapy should be added to lifestyle therapy. A

- For patients with diabetes aged $<40$ years with additional ASCVD risk factors, the patient and provider should consider using moderate-intensity statin in addition to lifestyle therapy. C

- For patients with diabetes aged 40-75 years $A$ and $>75$ years $B$ without ASCVD, use moderateintensity statin in addition to lifestyle therapy.

- In clinical practice, providers may need to adjust the intensity of statin therapy based on individual patient response to medication (e.g., side effects, tolerability, LDL cholesterol levels, or percent LDL reduction on statin therapy). For patients who do not tolerate the intended intensity of the statin, the maximally tolerated statin dose should be used. E

- For patients with diabetes and ASCVD, if LDL cholesterol is $\geq 70 \mathrm{mg} / \mathrm{dL}(3.9 \mathrm{mmol} / \mathrm{L})$ on maxi- 
mally tolerated statin dose, consider adding additional LDLlowering therapy (such as ezetimibe or PCSK9 inhibitor) after evaluating the potential for further ASCVD risk reduction, drug-specific adverse effects, and patient preferences. Ezetimibe may be preferred due to lower cost. A

- Statin therapy is contraindicated in pregnancy. B

- For patients with fasting triglyceride levels $\geq 500 \mathrm{mg} / \mathrm{dL}$ (5.7 $\mathrm{mmol} / \mathrm{L}$ ), evaluate for secondary causes of hypertriglyceridemia and consider medical therapy to reduce the risk of pancreatitis. $\mathrm{C}$

- Combination therapy (statin/ fibrate) has not been shown to improve ASCVD outcomes and is generally not recommended. A

- Combination therapy (statin/ niacin) has not been shown to provide additional cardiovascular benefit above statin therapy alone, may increase the risk of stroke with additional side effects, and is generally not recommended. A

Table 8 and Table 9 provide recommendations on high-intensity and moderate-intensity statin therapy.

\section{Antiplatelet Agents}

\section{Recommendations}

- Use aspirin therapy (75-162 $\mathrm{mg} /$ day) as a secondary prevention strategy in those with diabetes and a history of ASCVD. A

- For patients with ASCVD and documented aspirin allergy, clopidogrel (75 mg/day) should be used. B

- Dual antiplatelet therapy (with low-dose aspirin and a P2Y12 inhibitor) is reasonable for a year after an acute coronary syndrome $A$ and may have benefits beyond this period. $B$

- Aspirin therapy (75-162 mg/day) may be considered as a primary prevention strategy in those with type 1 or type 2 diabetes who are at increased cardiovascular risk. This includes most men and women with diabetes aged $\geq 50$ years who have at least one additional major risk factor (family history of premature ASCVD, hypertension, dyslipidemia, smoking, or albuminuria) and are not at increased risk of bleeding. C

\section{Coronary Heart Disease}

\section{Recommendations}

- In asymptomatic patients, routine screening for coronary artery disease is not recommended as it does not improve outcomes as long as ASCVD risk factors are treated. A

- Consider investigations for coronary artery disease in the presence of any of the following: atypical cardiac symptoms (e.g., unexplained dyspnea, chest discomfort); signs or symptoms of associated vascular disease including carotid bruits, transient ischemic attack, stroke, claudication, or PAD; or electrocardiogram abnormalities (e.g., Q waves). E

- In patients with known ASCVD, consider ACE inhibitor or ARB therapy to reduce the risk of cardiovascular events. B

- In patients with type 2 diabetes with stable congestive heart failure, metformin may be used if eGFR remains $>30 \mathrm{~mL} / \mathrm{min}$ but should be avoided in unstable or hospitalized patients with congestive heart failure. B

- In patients with type 2 diabetes and established ASCVD, antihyperglycemic therapy should begin with lifestyle management and metformin and subsequently incorporate an agent proven to reduce major adverse cardiovascular events and cardiovascular mortality (currently empagliflozin and liraglutide), after considering

\section{TABLE 8. Recommendations for Statin and Combination Treatment in Adults With Diabetes}

\begin{tabular}{|c|c|c|}
\hline Age & ASCVD & $\begin{array}{l}\text { Recommended statin intensity } \wedge \text { and combination } \\
\text { treatment }{ }^{\star}\end{array}$ \\
\hline \multirow[t]{3}{*}{$<40$ years } & No & Nonet \\
\hline & Yes & High \\
\hline & & $\begin{array}{l}\text { - If LDL cholesterol } \geq 70 \mathrm{mg} / \mathrm{dL} \text { ( } 3.9 \mathrm{mmol} / \mathrm{L} \text { ) despite } \\
\text { maximally tolerated statin dose, consider adding } \\
\text { additional LDL-lowering therapy (such as ezetimibe } \\
\text { or PCSK9 inhibitor)\# }\end{array}$ \\
\hline \multirow[t]{3}{*}{$\geq 40$ years } & No & Moderate $\neq$ \\
\hline & Yes & High \\
\hline & & $\begin{array}{l}\text { - If LDL cholesterol } \geq 70 \mathrm{mg} / \mathrm{dL} \text { ( } 3.9 \mathrm{mmol} / \mathrm{L} \text { ) despite } \\
\text { maximally tolerated statin dose, consider adding } \\
\text { additional LDL-lowering therapy (such as ezetimibe } \\
\text { or PCSK9 inhibitor) }\end{array}$ \\
\hline
\end{tabular}

*In addition to lifestyle therapy. $\wedge$ For patients who do not tolerate the intended intensity of statin, the maximally tolerated statin dose should be used. TModerate-intensity statin may be considered based on risk-benefit profile and presence of ASCVD risk factors. ASCVD risk factors include $L D L$ cholesterol $\geq 100 \mathrm{mg} / \mathrm{dL}$ (2.6 mmol/L), high blood pressure, smoking, CKD, albuminuria, and family history of premature ASCVD. $¥$ High-intensity statin may be considered based on risk-benefit profile and presence of ASCVD risk factors. \#Adults aged $<40$ years with prevalent ASCVD were not well represented in clinical trials of nonstatin-based LDL reduction. Before initiating combination lipid-lowering therapy, consider the potential for further ASCVD risk reduction, drug-specific adverse effects, and patient preferences. 
TABLE 9. High-Intensity and Moderate-Intensity Statin Therapy*

High-intensity statin therapy

(lowers LDL cholesterol by $\geq 50 \%$ )

Atorvastatin 40-80 mg

Rosuvastatin 20-40 mg
Moderate-intensity statin therapy

(lowers LDL cholesterol by 30-50\%)

Atorvastatin 10-20 mg

Rosuvastatin 5-10 mg

Simvastatin 20-40 mg

Pravastatin 40-80 mg

Lovastatin $40 \mathrm{mg}$

Fluvastatin XL $80 \mathrm{mg}$

Pitavastatin 2-4 mg

*Once-daily dosing. $X L$, extended release.

drug-specific and patient factors (see Table 7). A

- In patients with type 2 diabetes and established ASCVD, after lifestyle management and metformin, the antihyperglycemic agent canagliflozin may be considered to reduce major adverse cardiovascular events, based on drug-specific and patient factors (see Table 7). C

\section{MICROVASCULAR COMPLICATIONS AND FOOT CARE}

\section{Diabetic Kidney Disease}

Diabetic kidney disease (DKD) is a clinical diagnosis usually made based on the presence of albuminuria and/or reduced eGFR in the absence of signs or symptoms of other primary causes of kidney damage. CKD is diagnosed by the persistent presence of elevated urinary albumin excretion (albuminuria), low eGFR, or other manifestations of kidney damage (Table 10). $\mathrm{DKD}$, or CKD attributed to diabetes, occurs in $20-40 \%$ of patients with diabetes. DKD typically develops after diabetes duration of 10 years in type 1 diabetes, but may be present at diagnosis of type 2 diabetes. DKD can progress to end-stage renal disease (ESRD) requiring dialysis or kidney transplantation and is the leading cause of ESRD in the United States. In addition, among people with type 1 or type 2 diabetes, the presence of CKD markedly increases cardiovascular risk.

\section{Recommendations}

Screening

- At least once a year, assess urinary albumin (e.g., spot UACR) and eGFR in patients with type 1 diabetes with duration of $\geq 5$ years, in all patients with type 2 diabetes, and in all patients with comorbid hypertension. $\mathrm{B}$

\section{Treatment}

- Optimize glucose control to reduce the risk or slow the progression of DKD. A

- Optimize blood pressure control to reduce the risk or slow the progression of DKD. A

- For people with nondialysis-dependent DKD, dietary protein intake should be approximately $0.8 \mathrm{~g} / \mathrm{kg}$ body weight per day (the recommended daily allowance). For patients on dialysis, higher levels of dietary protein intake should be considered. B

- In nonpregnant patients with diabetes and hypertension, either an ACE inhibitor or an ARB is recommended for those with modestly elevated UACR ratio $(30-299 \mathrm{mg} / \mathrm{g} \mathrm{Cr}$ ) B and is strongly recommended for those with UACR $\geq 300 \mathrm{mg} / \mathrm{g} \mathrm{Cr}$ and/or eGFR $<60 \mathrm{~mL} / \mathrm{min} / 1.73 \mathrm{~m}^{2}$. A

- An ACE inhibitor or an ARB is not recommended for the primary prevention of DKD in patients with diabetes who have normal blood pressure, normal UACR $(<30 \mathrm{mg} / \mathrm{g}$ $\mathrm{Cr}$ ), and normal eGFR. B
- When eGFR is $<60 \mathrm{~mL} / \mathrm{min} / 1.73$ $\mathrm{m}^{2}$, evaluate and manage potential complications of chronic kidney disease. E

- Patients should be referred for evaluation for renal replacement treatment if they have an eGFR $<30 \mathrm{~mL} / \mathrm{min} / 1.73 \mathrm{~m}^{2}$. A

- Promptly refer to a physician experienced in the care of kidney disease for uncertainty about the etiology of kidney disease, difficult management issues, and rapidly progressing kidney disease. $B$

\section{Interventions}

Specific Glucose-Lowering

Medications

A number of large cardiovascular outcomes trials in patients with type 2 diabetes at high risk for CVD or with existing CVD (EMPA-REG OUTCOME [BI 10773 (Empagliflozin) Cardiovascular Outcome Event Trial in Type 2 Diabetes Mellitus Patients], CANVAS [Canagliflozin Cardiovascular Assessment Study], LEADER [Liraglutide Effect and Action in Diabetes: Evaluation of Cardiovascular Outcome ResultsA Long Term Evaluation], and SUSTAIN-6 [Trial to Evaluate Cardiovascular and Other Longterm Outcomes With Semaglutide in Subjects With Type 2 Diabetes]) examined kidney effects as secondary outcomes with some positive results. Additional trials with primary kidney outcomes are needed to definitively determine whether other specific glucose-lowering drugs improve renal outcomes. See the full 2018 Standards of Care for additional discussion.

CVD and Blood Pressure

Hypertension is a strong risk factor for the development and progression of DKD. Antihypertensive therapy reduces the risk of albuminuria, and among patients with type 1 or type 2 diabetes with established DKD (eGFR $<60 \mathrm{~mL} / \mathrm{min} / 1.73 \mathrm{~m}^{2}$ and UACR $\geq 300 \mathrm{mg} / \mathrm{g} \mathrm{Cr}$ ), ACE inhibitor or ARB therapy reduces the risk of progression to ESRD. Moreover, 
TABLE 10. CKD Stages and Corresponding Focus of Kidney-Related Care

\begin{tabular}{|c|c|c|c|c|c|c|}
\hline \multicolumn{3}{|c|}{ CKD Staget } & \multicolumn{4}{|c|}{ Focus of Kidney-Related Care } \\
\hline Stage & $\begin{array}{c}\text { eGFR } \\
\left(\mathrm{ml} / \mathrm{min} / 1.73 \mathrm{~m}^{2}\right)\end{array}$ & $\begin{array}{l}\text { Evidence } \\
\text { of Kidney } \\
\text { Damage* }\end{array}$ & $\begin{array}{l}\text { Diagnose } \\
\text { Cause of } \\
\text { Kidney } \\
\text { Injury }\end{array}$ & $\begin{array}{l}\text { Evaluate and } \\
\text { Treat Risk } \\
\text { Factors for CKD } \\
\text { Progression** }\end{array}$ & $\begin{array}{l}\text { Evaluate and } \\
\text { Treat CKD } \\
\text { Complications }\end{array}$ & $\begin{array}{c}\text { Prepare } \\
\text { for Renal } \\
\text { Replacement } \\
\text { Therapy }\end{array}$ \\
\hline
\end{tabular}

No clinical

evidence of

CKD

\begin{tabular}{lcccccc}
\hline 1 & $\geq 90$ & + & $\checkmark$ & $\checkmark$ & & \\
\hline 2 & $60-89$ & + & $\checkmark$ & $\checkmark$ & $\checkmark$ & \\
\hline 3 & $30-59$ & $+/-$ & $\checkmark$ & $\checkmark$ & $\checkmark$ & $\checkmark$ \\
\hline 4 & $15-29$ & $+/-$ & & $\checkmark$ & $\checkmark$ & $\checkmark$ \\
\hline 5 & $<15$ & $+/-$ & & & $\checkmark$ & \\
\hline
\end{tabular}

TCKD stages 1 and 2 are defined by evidence of kidney damage (+), while CKD stages 3-5 are defined by reduced eGFR with or without evidence of kidney damage (+/-). *Kidney damage is most often manifest as albuminuria (UACR $\geq 30 \mathrm{mg} / \mathrm{g} \mathrm{Cr}$ ) but can also include glomerular hematuria, other abnormalities of the urinary sediment, radiographic abnormalities, and other presentations. ${ }^{*}$ Risk factors for CKD progression include elevated blood pressure, glycemia, and albuminuria. ${ }^{* \star}$ See Table 10.2 in the full 2018 Standards of Care.

antihypertensive therapy reduces risks of cardiovascular events.

Blood pressure levels < $140 / 90$ $\mathrm{mmHg}$ are generally recommended to reduce CVD mortality and slow CKD progression among people with diabetes. Lower blood pressure targets (e.g., <130/80 mmHg) may be considered for patients based on individual anticipated benefits and risks. ACE inhibitors or ARBs are the preferred first-line agent for blood pressure treatment among patients with diabetes, hypertension, eGFR $<60 \mathrm{~mL} / \mathrm{min} / 1.73 \mathrm{~m}^{2}$, and UACR $\geq 300 \mathrm{mg} / \mathrm{g}$ Cr because of their proven benefits for prevention of CKD progression. In general, $\mathrm{ACE}$ inhibitors and ARBs are considered to have similar benefits and risks. In the setting of lower levels of albuminuria $(30-299 \mathrm{mg} / \mathrm{g}$ Cr), ACE inhibitor or ARB therapy has been demonstrated to reduce progression to more advanced albuminuria $(\geq 300 \mathrm{mg} / \mathrm{g} \mathrm{Cr})$ and cardiovascular events but not progression to ESRD. While ACE inhibitors or $A R B s$ are often prescribed for albuminuria without hypertension, clinical trials have not been performed in this setting to determine whether this improves renal outcomes.

$A C E$ inhibitors or $A R B$ s are not recommended for patients without hypertension to prevent the development of DKD.

Two clinical trials studied the combinations of ACE inhibitors and $A R B s$ and found no benefits on CVD or DKD, and the drug combination had higher adverse event rates (hyperkalemia and/or acute kidney injury). Therefore, the combined use of ACE inhibitors and ARBs should be avoided.

\section{Diabetic Retinopathy}

\section{Recommendations}

- Optimize glycemic control to reduce the risk or slow the progression of diabetic retinopathy. A

- Optimize blood pressure and serum lipid control to reduce the risk or slow the progression of diabetic retinopathy. A

\section{Screening}

- Adults with type 1 diabetes should have an initial dilated and comprehensive eye examination by an ophthalmologist or optometrist within 5 years after the onset of diabetes. B
- Patients with type 2 diabetes should have an initial dilated and comprehensive eye examination by an ophthalmologist or optometrist at the time of the diabetes diagnosis. B

- If there is no evidence of retinopathy for one or more annual eye exam and glycemia is well controlled, then exams every 1-2 years may be considered. If any level of diabetic retinopathy is present, subsequent dilated retinal examinations should be repeated at least annually by an ophthalmologist or optometrist. If retinopathy is progressing or sight-threatening, then examinations will be required more frequently. B

- While retinal photography may serve as a screening tool for retinopathy, it is not a substitute for a comprehensive eye exam. E

- Women with preexisting type 1 or type 2 diabetes who are planning pregnancy or who are pregnant should be counseled on the risk of development and/or progression of diabetic retinopathy. $B$

- Eye examinations should occur before pregnancy or in the first trimester in patients with preexisting type 1 or type 2 diabetes, 
and then patients should be monitored every trimester and for 1 year postpartum as indicated by the degree of retinopathy. $B$

Treatment

- Promptly refer patients with any level of macular edema, severe nonproliferative diabetic retinopathy (a precursor of proliferative diabetic retinopathy), or any proliferative diabetic retinopathy to an ophthalmologist who is knowledgeable and experienced in the management of diabetic retinopathy. A

- The traditional standard treatment, panretinal laser photocoagulation therapy, is indicated to reduce the risk of vision loss in patients with high-risk proliferative diabetic retinopathy and, in some cases, severe nonproliferative diabetic retinopathy. A

- Intravitreous injections of antivascular endothelial growth factor ranibizumab are not inferior to traditional panretinal laser photocoagulation and are also indicated to reduce the risk of vision loss in patients with proliferative diabetic retinopathy. A

- Intravitreous injections of antivascular endothelial growth factor are indicated for central-involved diabetic macular edema, which occurs beneath the foveal center and may threaten reading vision. A

- The presence of retinopathy is not a contraindication to aspirin therapy for cardioprotection, as aspirin does not increase the risk of retinal hemorrhage. A

\section{Neuropathy}

\section{Recommendations}

Screening

- All patients should be assessed for diabetic peripheral neuropathy starting at diagnosis of type 2 diabetes and 5 years after the diagnosis of type 1 diabetes and at least annually thereafter. B

- Assessment for distal symmetric polyneuropathy should include a careful history and assessment of either temperature or pinprick sensation (small-fiber function) and vibration sensation using a $128-\mathrm{Hz}$ tuning fork (for largefiber function). All patients should have annual $10-\mathrm{g}$ monofilament testing to identify feet at risk for ulceration and amputation. B

- Symptoms and signs of autonomic neuropathy should be assessed in patients with microvascular complications. E

\section{Treatment}

- Optimize glucose control to prevent or delay the development of neuropathy in patients with type 1 diabetes $A$ and to slow the progression of neuropathy in patients with type 2 diabetes. B

- Assess and treat patients to reduce pain related to diabetic peripheral neuropathy B and symptoms of autonomic neuropathy and to improve quality of life. E

- Either pregabalin or duloxetine are recommended as initial pharmacologic treatments for neuropathic pain in diabetes. A

The diabetic neuropathies are a diagnosis of exclusion and are a heterogeneous group of disorders with diverse clinical manifestations. The early recognition and appropriate management of neuropathy in the patient with diabetes is important.

\section{Diagnosis}

Diabetic Peripheral Neuropathy The following clinical tests may be used to assess small- and large-fiber function and protective sensation:

1. Small-fiber function: pinprick and temperature sensation

2. Large-fiber function: vibration perception and 10-g monofilament

3. Protective sensation: 10 -g monofilament

These tests not only screen for the presence of dysfunction but also predict future risk of complications. Electrophysiological testing or refer- ral to a neurologist is rarely needed, except in situations where the clinical features are atypical or the diagnosis is unclear. See the ADA position statement "Diabetic Neuropathy" for more details.

\section{Foot Care}

\section{Recommendations}

- Perform a comprehensive foot evaluation at least annually to identify risk factors for ulcers and amputations. B

- All patients with diabetes should have their feet inspected at every visit. C

- Obtain a prior history of ulceration, amputation, Charcot foot, angioplasty or vascular surgery, cigarette smoking, retinopathy, and renal disease and assess current symptoms of neuropathy (pain, burning, numbness) and vascular disease (leg fatigue, claudication). B

- The examination should include inspection of the skin, assessment of foot deformities, neurological assessment (10-g monofilament testing with at least one other assessment: pinprick, temperature, vibration), and vascular assessment including pulses in the legs and feet. $B$

- Patients with symptoms of claudication or decreased or absent pedal pulses should be referred for ankle-brachial index and for further vascular assessment as appropriate. C

- A multidisciplinary approach is recommended for individuals with foot ulcers and high-risk feet (e.g., dialysis patients and those with Charcot foot, prior ulcers, or amputation). B

- Refer patients who smoke or who have histories of prior lowerextremity complications, loss of protective sensation, structural abnormalities, or PAD to foot care specialists for ongoing preventive care and life-long surveillance. $C$

- The use of specialized therapeutic footwear is recommended for 
high-risk patients with diabetes including those with severe neuropathy, foot deformities, or history of amputation. B

\section{OLDER ADULTS}

\section{Recommendations}

- Consider the assessment of medical, psychological, functional, and social geriatric domains in older adults to provide a framework to determine targets and therapeutic approaches for diabetes management. C

- Screening for geriatric syndromes may be appropriate in older adults experiencing limitations in their basic and instrumental activities of daily living as they may affect diabetes self-management and be related to health-related quality of life. C

- Screening for early detection of mild cognitive impairment or dementia and depression is indicated for adults 65 years of age or older at the initial visit and annually as appropriate. B

- Hypoglycemia should be avoided in older adults with diabetes. It should be assessed and managed by adjusting glycemic targets and pharmacologic interventions. B

- Older adults who are otherwise healthy with few coexisting chronic illnesses and intact cognitive function and functional status should have lower glycemic goals (A1C < $7.5 \%$ [58 $\mathrm{mmol} / \mathrm{mol}]$ ), while those with multiple coexisting chronic illnesses, cognitive impairment, or functional dependence should have less stringent glycemic goals (A1C $<8.0-8.5 \%$ [64-69 $\mathrm{mmol} / \mathrm{mol}]) . \mathrm{C}$

- Glycemic goals for some older adults might reasonably be relaxed as part of individualized care, but hyperglycemia leading to symptoms or risk of acute hyperglycemic complications should be avoided in all patients. C

- Screening for diabetes complications should be individualized in older adults. Particular attention should be paid to complications that would lead to functional impairment. C

- Treatment of hypertension to individualized target levels is indicated in most older adults. C

- Treatment of other cardiovascular risk factors should be individualized in older adults considering the time frame of benefit. Lipidlowering therapy and aspirin therapy may benefit those with life expectancies at least equal to the time frame of primary prevention or secondary intervention trials. E

- Consider diabetes education for the staff of long-term care facilities to improve the management of older adults with diabetes. E

- Patients with diabetes residing in long-term care facilities need careful assessment to establish glycemic goals and to make appropriate choices of glucose-lowering agents based on their clinical and functional status. E

Diabetes is an important health condition for the aging population as approximately one-quarter of people over the age of 65 years have diabetes. Older individuals with diabetes have higher rates of premature death, functional disability, and coexisting illness.

People with diabetes have higher incidences of all-cause dementia, Alzheimer disease, and vascular dementia than people with normal glucose tolerance. Poor glycemic control is associated with decline in cognitive function.

It is important to prevent hypoglycemia to reduce the risk of cognitive decline and other major adverse outcomes. Older adults are at higher risk of hypoglycemia for many reasons, including insulin deficiency necessitating insulin therapy and progressive renal insufficiency. Hypoglycemic events should be diligently monitored and avoided, whereas glycemic targets and pharmacologic interventions may need to be adjusted to accommodate for the changing needs of the older adult.
The care of older adults with diabetes is complicated by their clinical, cognitive, and functional heterogeneity. Providers caring for older adults with diabetes should prioritize treatment goals (Table 11). For patients with complications and reduced functionality, it is reasonable to set less intensive glycemic goals. Factors to consider in individualizing glycemic goals are outlined in Figure 1.

\section{Pharmacologic Therapy}

Special care is required in prescribing and monitoring pharmacologic therapy in older adults (see Table 7). The patient's living situation must be considered because it may affect diabetes management and support. When patients are found to have a regimen beyond their self-management abilities, treatment should be deintensified (or simplified) to reduce hypoglycemia and disease-related distress while avoiding a substantial worsening of glycemic control.

Older adults with diabetes are likely to benefit from control of other cardiovascular risk factors. Evidence is strong for treatment of hypertension. There is less evidence for lipid-lowering and aspirin therapy, although the benefits of these interventions are likely to apply to older adults whose life expectancies equal or exceed the time frames of clinical prevention trials.

\section{Treatment in Skilled Nursing Facilities and Nursing Homes}

Management of diabetes is unique in the long-term care (LTC) setting (i.e., nursing homes and skilled nursing facilities). Individualization of health care is important for all patients. For patients in the LTC setting, special attention should be given to nutritional considerations, end-of-life care, and changes in diabetes management with respect to advanced disease. Withdrawal of medications may be appropriate. For more information, see the ADA position statement "Management of Diabetes in Long-Term Care and Skilled Nursing Facilities." 


\section{CHILDREN AND ADOLESCENTS}

See the full 2018 Standards of Care regarding the treatment of children with type 1 diabetes and for information on hypertension and dyslipidemia in children.

\section{Glycemic Control}

Recommendations

- An A1C goal of $<7.5 \%$ (58 mmol/ $\mathrm{mol}$ ) is recommended across all pediatric age-groups. E

\section{Type 2 Diabetes}

In the past 20 years, the incidence and prevalence of type 2 diabetes in youth has increased, especially in racial and ethnic minority populations. Evidence suggests that type 2 diabetes in youth is different not only from type 1 diabetes but also from type 2 diabetes in adults and has unique features, such as a more rapidly progressive decline in $\beta$-cell function and accelerated development of diabetes complications. Risk factors associated with type 2 diabetes in youth include, in addition to racial/ethnic minority group status, adiposity, family history of diabetes, female sex, and low socioeconomic status.

\section{Recommendations}

Screening and Diagnosis

- Risk-based screening for prediabetes and/or type 2 diabetes should be considered in children and adolescents after the onset of puberty or $\geq 10$ years of age, whichever occurs earlier, who are overweight (BMI $>85$ th \%) or obese $($ BMI $>95$ th \%) and who have one or more additional risk factors for diabetes (see Table 4). A

- If tests are normal, repeat testing at a minimum of 3-year intervals $\mathrm{E}$, or more frequently if $\mathrm{BMI}$ is increasing. $\mathrm{C}$

\section{Pharmacologic Management}

- Initiate pharmacologic therapy, in addition to lifestyle therapy, at diagnosis of type 2 diabetes. A

- In metabolically stable patients (A1C $<8.5 \%$ and asymptomatic), metformin is the initial phar- macologic treatment of choice if renal function is $>30 \mathrm{~mL} / \mathrm{min} /$ $1.73 \mathrm{~m}^{2} . \mathrm{A}$

- Youth with marked hyperglycemia (blood glucose $\geq 250 \mathrm{mg} / \mathrm{dL}$ [13.9 $\mathrm{mmol} / \mathrm{L}], \mathrm{A} 1 \mathrm{C} \geq 8.5 \%$ [69 $\mathrm{mmol} / \mathrm{mol}]$ ) without ketoacidosis at diagnosis who are symptomatic with polyuria, polydipsia, nocturia, and/or weight loss should be treated initially with basal insulin while metformin is initiated and titrated to maximally tolerated dose to achieve A1C goal. E

- When the A1C target is no longer met with metformin monotherapy, or if contraindications or intolerable side effects of metformin develop, basal insulin therapy should be initiated. E

- In patients initially treated with basal insulin and metformin who are meeting glucose targets based on home blood glucose monitoring, basal insulin can be tapered over $2-6$ weeks by decreasing the insulin dose by $10-30 \%$ every few days. A

\section{MANAGEMENT OF DIABETES IN PREGNANCY}

The majority of diabetes in pregnancy is GDM, with the remainder primarily preexisting type 1 diabetes and type 2 diabetes. Good preconception care for women with existing diabetes has been shown to dramatically improve outcomes for both mother and child.

\section{Preconception Counseling}

\section{Recommendations}

- Starting at puberty, preconception counseling should be incorporated into routine diabetes care for all girls of childbearing potential. A

- Family planning should be discussed and effective contraception should be prescribed and used until a woman is prepared and ready to become pregnant. $A$

- Preconception counseling should address the importance of glycemic control as close to normal as is safely possible, ideally $\mathrm{A} 1 \mathrm{C}<6.5 \%$
(48 $\mathrm{mmol} / \mathrm{mol}$ ), to reduce the risk of congenital anomalies. B

- Women with preexisting type 1 or type 2 diabetes who are planning pregnancy or who have become pregnant should be counseled on the risk of development and/or progression of diabetic retinopathy. Dilated eye examinations should occur before pregnancy or in the first trimester, and then patients should be monitored every trimester and for 1 year postpartum as indicated by the degree of retinopathy and as recommended by the eye care provider. $B$

See the full 2018 Standards of Care for more information on management of diabetes during pregnancy.

\section{Postpartum Care}

Postpartum care should include psychosocial assessment and support for self-care.

Women with GDM should be tested for persistent diabetes or prediabetes at 4-12 weeks postpartum with a 75-g OGTT using nonpregnancy criteria. Women should also be tested every 1-3 years thereafter if the 4- to 12-week 75-g OGTT is normal. Ongoing evaluation may be performed with any recommended glycemic test (e.g., A1C, FPG, or 75-g OGTT using nonpregnant thresholds).

In women with preexisting type 1 or type 2 diabetes taking insulin, particular attention should be directed to hypoglycemia prevention in the setting of breastfeeding and erratic sleep and eating schedules.

All women with diabetes of childbearing potential should have family planning options reviewed at regular intervals.

\section{DIABETES CARE IN THE HOSPITAL}

Hospitals should promote the shortest safe hospital stay and provide an effective transition out of the hospital that prevents acute complications and readmission. Prevention of hypogly- 


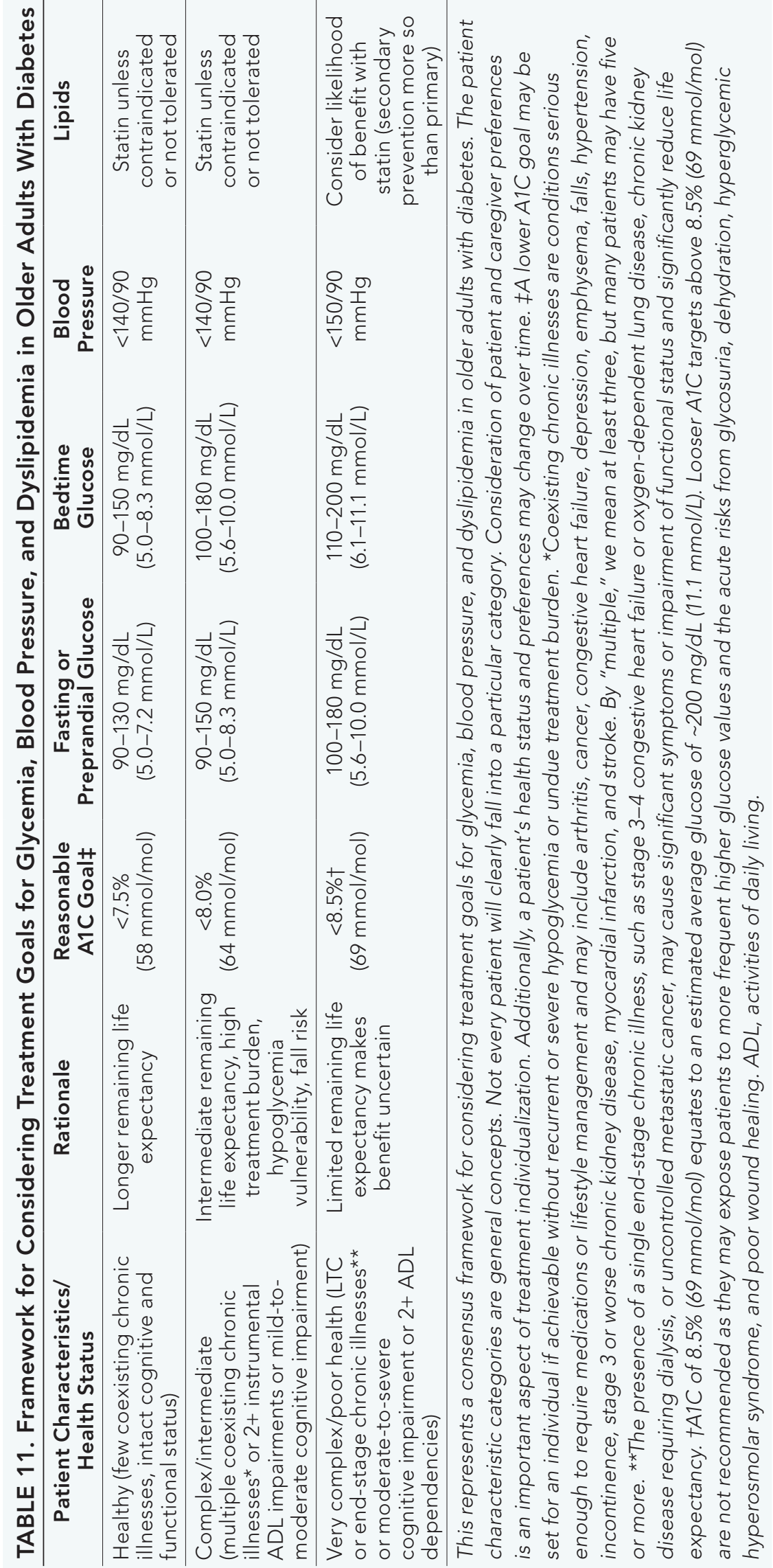

cemia and hyperglycemia should be goals, since adverse outcomes are associated with both.

\section{Hospital Care Delivery Standards}

\section{Recommendation}

- Perform an A1C on all patients with diabetes or hyperglycemia (blood glucose $>140 \mathrm{mg} / \mathrm{dL}$ ) admitted to the hospital if not performed in the prior 3 months. B

\section{Considerations on Admission}

Diabetes self-management should be assessed on admission in addition to A1C.

\section{Physician Order Entry}

Best practice care can often be ensured by the use of structured order sets consistent with quality assurance standards.

\section{Recommendation}

- Insulin should be administered using validated written or computerized protocols that allow for predefined adjustments in the insulin dosage based on glycemic fluctuations. E

\section{Glycemic Targets in Hospitalized Patients}

\section{Recommendations}

- Insulin therapy should be initiated for treatment of persistent hyperglycemia starting at a threshold $\geq 180 \mathrm{mg} / \mathrm{dL}(10.0 \mathrm{mmol} / \mathrm{L})$. Once insulin therapy is started, a target glucose range of $140-180$ $\mathrm{mg} / \mathrm{dL}(7.8-10.0 \mathrm{mmol} / \mathrm{L})$ is recommended for the majority of critically ill patients and noncritically ill patients. A

- More stringent goals, such as 110 $140 \mathrm{mg} / \mathrm{dL}$ (6.1-7.8 mmol/L), may be appropriate for selected patients, if this can be achieved without significant hypoglycemia. C

Hyperglycemia in hospitalized patients is defined as blood glucose levels $>140 \mathrm{mg} / \mathrm{dL}(7.8 \mathrm{mmol} / \mathrm{L})$. The hypoglycemia alert value in hospitalized patients is defined as blood glucose $\leq 70 \mathrm{mg} / \mathrm{dL}(3.9 \mathrm{mmol} / \mathrm{L})$ 
and clinically significant hypoglycemia as glucose values $<54 \mathrm{mg} / \mathrm{dL}(3.0$ $\mathrm{mmol} / \mathrm{L}$ ).

\section{Bedside Blood Glucose Monitoring}

In the patient who is eating meals, glucose monitoring should be performed before meals. In the patient who is not eating, glucose monitoring is advised every 4-6 h. Testing every $30 \mathrm{~min}$ to $2 \mathrm{~h}$ is required for intravenous insulin infusion.

Glucose results from a POC meter that do not correlate with the patient's clinical status should be confirmed with conventional laboratory measures. Several inpatient studies have shown that CGM use did not improve glucose control but detected a greater number of hypoglycemic events than POC testing. However, a recent review has recommended against using CGM in adults in a hospital setting until more safety and efficacy data become available.

\section{Antihyperglycemic Agents in Hospitalized Patients}

\section{Recommendations}

- A basal plus bolus correction insulin regimen, with the addition of nutritional insulin in patients who have good nutritional intake, is the preferred treatment for noncritically ill patients. A

- Sole use of sliding scale insulin in the inpatient hospital setting is strongly discouraged. A

In most instances in the hospital setting, insulin is the preferred treatment for glycemic control. However, in certain circumstances, it may be appropriate to continue home regimens including oral antihyperglycemic medications.

\section{Insulin Therapy}

In the critical care setting, continuous intravenous insulin infusion has been shown to be the best method for achieving glycemic targets. Outside of critical care units, scheduled insulin regimens as described above are recommended.
If the patient is eating, insulin injections should align with meals. In such instances, POC glucose testing should be performed immediately before meals. Type 1 diabetes patients should have basal-bolus plus nutritional insulin if the patient is eating. A transition protocol from insulin infusion to subcutaneous insulin is recommended.

\section{Noninsulin Therapies}

The safety and efficacy of noninsulin antihyperglycemic therapies in the hospital setting is an area of active research. See the full 2018 Standards of Care for a comprehensive review of the inpatient use of these medications.

\section{Hypoglycemia}

\section{Recommendations}

- A hypoglycemia management protocol should be adopted and implemented by each hospital or hospital system. A plan for preventing and treating hypoglycemia should be established for each patient. Episodes of hypoglycemia in the hospital should be documented in the medical record and tracked. E

- The treatment regimen should be reviewed and changed as necessary to prevent further hypoglycemia when a blood glucose value is $\leq 70$ $\mathrm{mg} / \mathrm{dL}(3.9 \mathrm{mmol} / \mathrm{L})$. C

While hypoglycemia is associated with increased mortality, hypoglycemia may be a marker of underlying disease rather than the cause of increased mortality. However, until it is proven not to be causal, it is prudent to avoid hypoglycemia.

Iatrogenic triggers should be considered. Studies of "bundled" preventative therapies including proactive surveillance of glycemic outliers and an interdisciplinary data-driven approach to glycemic management showed that hypoglycemic episodes in the hospital could be prevented.

\section{MNT in the Hospital}

The goals of MNT are to optimize glycemic control, provide adequate calories to meet metabolic demands, and address personal food preferences. The ADA does not endorse any single meal plan. A registered dietitian can serve as an inpatient team member.

\section{Self-Management in the Hospital}

Diabetes self-management in the hospital may be appropriate for select youth and adult patients. Sufficient cognitive and physical skills, adequate oral intake, proficiency in carbohydrate estimation, and knowledge of sick-day management are some of the requirements. See the full 2018 Standards of Care regarding selfadministered insulin with an MDI or CSII (insulin pump) regimen. A protocol should exist for these situations.

Standards for Special Situations See the full 2018 Standards of Care for guidance on enteral/parenteral feedings, diabetic ketoacidosis and hyperosmolar hyperglycemic state, perioperative care, and glucocorticoid therapy.

\section{Transition From the Acute Care Setting}

\section{Recommendation}

- There should be a structured discharge plan tailored to the individual patient with diabetes. $\mathrm{B}$

Tailor a structured discharge plan beginning at admission and update as patient needs change. It is important that patients be provided with appropriate durable medical equipment, medication reconciliation, supplies, and prescriptions, along with appropriate education at the time of discharge. An outpatient follow-up visit within 1 month of discharge is advised for all patients who have hyperglycemia in the hospital. Continuing contact may also be needed. Clear communication with outpatient providers either directly or via structured hospital discharge summaries facilitates safe transitions to outpatient care. If oral 
medications are held in the hospital, there should be protocols for resuming them 1-2 days before discharge.

Refer to the full 2018 Standards of Care for a complete discussion of readmission prevention.

\section{Diabetes Advocacy}

For a list of ADA advocacy position statements, including "Diabetes and Driving" and "Diabetes and Employment," see the Diabetes Advocacy section of the full 2018 Standards of Care.

\section{Acknowledgments}

This abridged version of the Standards of Medical Care in Diabetes - 2018 was created by the ADA's Primary Care Advisory Group (PCAG), with special thanks to PCAG chair Jay Shubrook, DO, of Vallejo, CA; PCAG vice-chair Eric L. Johnson, MD, of Grand Forks, ND; Amy Butts, PA-C, MPAS, CDE, of Weirton, WV; Sandra Leal, PharmD, MPH, FAPhA, CDE, of Tucson, AZ; Andrew S. Rhinehart, MD, FACP, FACE, CDE, BC-ADM, CDTC, of Marco Island, FL; and Neil Skolnik, MD, of Jenkintown, PA, with ADA staff support from Sarah Bradley.
The full Standards of Medical Care in Diabetes - 2018 was developed by the ADA's Professional Practice Committee: Rita R. Kalyani, MD, MHS, FACP (Chair), Christopher Cannon, MD, Andrea L. Cherrington, MD, MPH,* Donald R. Coustan, MD, Ian de Boer, MD, MS, ${ }^{*}$ Hope Feldman, CRNP, FNP-BC, Judith Fradkin, MD, David Maahs, MD, PhD, Melinda Maryniuk, Med, RD, CDE, Medha N. Munshi, MD,* Joshua J. Neumiller, PharmD, CDE, FASCP, and Guillermo E. Umpierrez.* ADA staff support includes Erika Gebel Berg, PhD, Tamara Darsow, $\mathrm{PhD}$, Matt Petersen, Sacha Uelmen, RDN, CDE, and William T. Cefalu, MD. (*Subgroup leaders) 